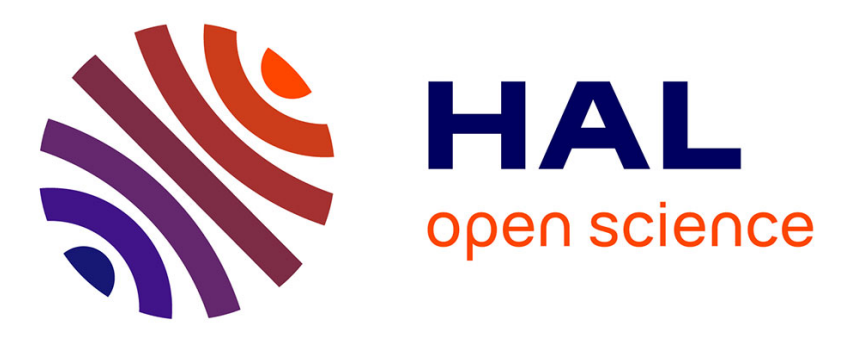

\title{
A Diffusion-Based Method for Long-T2 Suppression in Steady State Sequences: Validation and Application for 3D-UTE Imaging
}

Lucas Soustelle, Julien Lamy, François Rousseau, Jean-Paul Armspach, Paulo Loureiro de Sousa

\section{To cite this version:}

Lucas Soustelle, Julien Lamy, François Rousseau, Jean-Paul Armspach, Paulo Loureiro de Sousa. A Diffusion-Based Method for Long-T2 Suppression in Steady State Sequences: Validation and Application for 3D-UTE Imaging. Magnetic Resonance in Medicine, 2018, 80 (2), pp.548-559. 10.1002/mrm.27057 . hal-02285913

\section{HAL Id: hal-02285913 https://imt-atlantique.hal.science/hal-02285913}

Submitted on 16 Sep 2019

HAL is a multi-disciplinary open access archive for the deposit and dissemination of scientific research documents, whether they are published or not. The documents may come from teaching and research institutions in France or abroad, or from public or private research centers.
L'archive ouverte pluridisciplinaire HAL, est destinée au dépôt et à la diffusion de documents scientifiques de niveau recherche, publiés ou non, émanant des établissements d'enseignement et de recherche français ou étrangers, des laboratoires publics ou privés. 


\section{A diffusion-based method for long- $\mathrm{T}_{2}$ suppression in steady state sequences: Validation and application for 3D-UTE imaging}

Lucas Soustelle $^{1}$, Julien Lamy ${ }^{1}$, François Rousseau ${ }^{2}$, Jean-Paul Armspach ${ }^{1}$, Paulo Loureiro de Sousa ${ }^{1}$

1. Université de Strasbourg, CNRS, ICube, FMTS, Strasbourg, France

2. Institut Mines Télécom Atlantique, INSERM, LaTIM, Brest, France

Correspondence to:

Paulo Loureiro de Sousa

Institut de Physique Biologique

Université de Strasbourg, ICube, CNRS, FMTS

4 Rue Kirschleger, 67085 Strasbourg, France

ploureiro@unistra.fr

Running Head: Diffusion-based method for long- $T_{2}$ suppression in steady state 3D-UTE

Submitted to Magnetic Resonance in Medicine

The first author wishes the manuscript to be considered for the Young Investigator Award program.

Text word count: $5378 / 5000$ 


\section{Abstract}

Purpose: To introduce a novel method for long- $T_{2}$ signal physical suppression in steadystate based on configuration states combination and modulation using diffusion weighting. Its efficiency in yielding a high contrast in short- $T_{2}$ structures using an ultrashort echo time acquisition module (Diff-UTE) is compared to the adiabatically prepared InversionRecovery-UTE sequence (IR-UTE).

Theory and methods: Using a rectangular-pulse prepared 3D-UTE sequence, the possibility of long- $T_{2}$ component signal cancellation through diffusion effects is addressed, and the condition met for sets of sequence parameters. Simultaneously, the short- $T_{2}$ component signal is maximized using a Bloch equation-based optimization process. The method is evaluated from simulations, and experiments are conducted on a phantom composed of short and long- $T_{2}$ components, as well as on an ex vivo mouse head.

Results: Within equal scan times, the proposed method allowed for an efficient long- $T_{2}$ signal suppression, and expectedly yielded a higher signal to noise ratio in short- $T_{2}$ structures compared to the IR-UTE technique, although an intrinsic short- $T_{2}$ signal loss is expected through the preparation module.

Conclusion: The Diff-UTE method represents an interesting alternative to the IR-UTE technique. Diffusion weighting allowing for a long- $T_{2}$ suppression results in a less penalizing method to generate a high and selective contrast in short- $T_{2}$ components.

Keywords: ultrashort echo time (UTE); steady state; diffusion; long-T2 suppression; shortT2 contrast; preclinical

\section{Abstract word count: 196/200}




\section{INTRODUCTION}

Ultrashort echo time (UTE) MRI methods are used to acquire and quantify NMR signals from tissues with sub-millisecond transverse relaxation time $\left(T_{2}\right)$. Among them, one can cite cortical bone (1, 2, 3, 4) with strong perspective for CT-like use (5, 6, 7), meniscus (8, 9), tendon (10, 11, 12, 13), lung parenchyma (14, 15, 16), and myelin (17, 18, 19). Since UTE images are mainly proton-density weighted, an efficient method for suppressing water and fat signals (referred hereafter to as long- $T_{2}$ components $\left(T_{2}>10 \mathrm{~ms}\right)$ ) is commonly used with an UTE acquisition scheme to properly exhibit a positive contrast over the solid-like tissues of interest. Several schemes for long- $T_{2}$ signal suppression or attenuation have been proposed, such as saturation modules (20, 21), dual echo subtraction (22, 23), or even specific excitation patterns (24) to either null the undesired signal or to selectively excite the component of interest. One of the most frequently used methods to achieve an efficient suppression consists in using an inversion-recovery module in pair with an appropriate inversion time $(T I)$ to

physically null the long- $T_{2}$ component, followed by an UTE acquisition (22, 25). The IR-UTE sequence has often been adapted into enhanced versions for varied purposes (suppressing fat and water signal (20, 26, 27), filtering short- $T_{2}$ signals (28), and acceleration (29)), and has already demonstrated promising results in various applications (3, 19, 27, 30, 31).

Nevertheless, the IR-UTE sequence shows limitations in terms of short- $T_{2}$ signal to noise ratio (SNR) generation. Through the use of adiabatic inversion pulses that often saturate the short- $T_{2}$ components $(21,32)$, the available magnetization in the component of interest is governed by its longitudinal recovery during the inversion time delay, itself depending on the $T_{1}$ value of the component to be suppressed. Another issue that has to be considered for clinical applications is the elevated Specific Absorption Rate (SAR) induced by the use of adiabatic inversion pulses for long- $T_{2}$ signal suppression and short- $T_{2}$ signal filtering (28), especially for currently developing ultra-high field scanners.

This study presents a novel method for long- $T_{2}$ suppression in a steady-state 3D-UTE sequence through the use of diffusion weighting induced by spoiling gradients, while acquiring 
maximized short- $T_{2}$ signals (33). The behavior of the method was evaluated from simulations and phantom experiments as a proof of concept on a preclinical scanner. Comparison of the proposed sequence to the IR-UTE sequence was conducted on the same phantom and on an ex vivo mouse head.

\section{THEORY}

In the following, the superscripts ${ }^{L}$ and ${ }^{S}$ will indicate the long and short- $T_{2}$ component, respectively. Simulated signal relative amplitude will be introduced as $S=\left|M_{x y}\right| / M_{0}$, with $M_{x y}$ transverse magnetization, and $M_{0}$ its corresponding magnetization amplitude at thermal equilibrium. To provide a distinction between the two components having respective unknown proton density, it is further quantified in $\% M_{0}$.

\section{Diff-UTE sequence}

The employed pulse sequence (Fig. 1.a) consists in a long $\left(>T_{2}^{S}\right)$ rectangular preparation pulse followed by a short one, whose flip angle is computed to maximize the short- $T_{2}$ signal. In steady-state, the approach is somehow similar to the Actual Flip Angle method (34), except that the two consecutive pulses have different flip angles $\left(\alpha_{1} \neq \alpha_{2}\right)$ and durations, and the readout is performed exclusively after the second pulse. Hence, gradient spoiling, RF spoiling, and delays are optimized to ensure a steady-state of the long- $T_{2}$ component to be suppressed, as well as minimizing the impact of potential static gradients (e.g. $B_{0}$ inhomogeneities) (35). The sequence aims at removing the long- $T_{2}$ signal, while simultaneously maximizing the short- $T_{2}$ one, as described in the following sections.

\section{Short- $T_{2}$ signal description}

Considering the signal of interest arising from the short- $T_{2}$ component being fully spoiled before each excitation, its quantity can be tracked using the Bloch equations in steady-state 
immediately after the readout pulse $(T E=0 \mathrm{~ms})$ :

$$
S^{S}=\frac{\left(1-E_{s}\right)+E_{s}\left(1-E_{w}\right) f_{z_{1}}}{1-f_{z_{1}} f_{z_{2}} E_{s} E_{w}} f_{x y}
$$

with $E_{s}=e^{-T R_{1} / T_{1}^{S}}, E_{w}=e^{-T R_{2} / T_{1}^{S}}\left(T R_{1}\right.$ and $T R_{2}$ referring to the internal delays after

excitations), $f_{z_{i}}=e^{-w_{i}}\left[\cos \left(\gamma_{i}\right)+w_{i} \operatorname{sinc}\left(\gamma_{i}\right)\right], f_{x y}=e^{-w_{2}} \alpha_{2} \operatorname{sinc}\left(\gamma_{2}\right)\left(\right.$ with $\gamma_{i}=\sqrt{\alpha_{i}^{2}-w_{i}^{2}}$ and $\left.w_{i}=\tau_{i} / 2 T_{2}^{S}\right), \alpha_{i}$ and $\tau_{i}$ being the respective flip angles and pulse durations. A detailed derivation of the Bloch equations solutions is given in the Appendix. The functions $f_{z_{i}}$ and $f_{x y}$ account for the signal loss during a hard RF pulse caused by $T_{2}^{S}$ relaxation (mapping functions), as described in (36, 37, 38). Including the mapping functions is advantageous and helps quantifying the impact of the durations of the consecutive pulses (22, 39). It therefore allows to adequately model the available $S^{S}$ signal. Thus, once delays and pulses characteristics are set, the actual short- $T_{2}$ signal can be maximized with respect to $\alpha_{2}$ as $\hat{\alpha_{2}}=\operatorname{argmax}_{\alpha_{2}}\left(S^{S}\right)$.

\section{Long- $T_{2}$ signal description}

Once sequence parameters are set, we make use of the steady-state condition in a periodic MR sequence to suppress the water signal. The long- $T_{2}$ signal can be modeled by any simulation framework taking diffusion into account (40, 41). Thus, using the expression for configuration states in (42), the signal to be suppressed can be written:

$$
F_{0}^{+}=\cos ^{2}\left(\alpha_{2} / 2\right) F_{0}^{-}+e^{2 i \Phi} \sin ^{2}\left(\alpha_{2} / 2\right)^{2} F_{0}^{-*}-i e^{i \Phi} \sin \left(\alpha_{2}\right) Z_{0}^{-},
$$

with $F_{0}^{-}$and $Z_{0}^{-}$being functions of $\alpha_{1}, \alpha_{2}$, RF-phase $\Phi, T R_{1}, T R_{2}, T_{1}^{L}, T_{2}^{L}$, gradient spoiling areas $G \delta$ and $G \delta^{\prime}$ in respective internal segments $T R_{1}$ and $T R_{2}$, and tissue diffusion characteristics. In this case, having $\left|F_{0}^{+}\right|=0$ would imply a complete long- $T_{2}$ suppression. Since no trivial analytical expression exists for the $F_{0}^{-}$and $Z_{0}^{-}$states in steady-state, we numerically explored the tissues and sequence parameter space in order to assess whether 
the diffusion weighting induced by the spoiling gradients would combine the $F_{0}^{-}$and $Z_{0}^{-}$

states in order to satisfy $\left|F_{0}^{+}\right|=0$. This condition can be met for sets of parameters and RF-phase increment $\Phi_{0}=k \times 360 /(n+1)\left(k \in \mathbb{Z}, n=T R_{2} / T R_{1} \geq 2\right)$, offering a signal falling to 0 (referred hereafter to as "signal minimum").

In the following, the apparent diffusion coefficient $D$ will be assimilated to that of the single spoiling direction used in experiments (i.e. z-direction). Hence, and for better readability, diffusion weighting is quantified in terms of gradient spoiling moment $G \delta$, equal to the gradient area in the first internal delay $T R_{1}$. To maintain a steady-state condition, the spoiling gradient areas ratio $G \delta^{\prime} / G \delta$ is set to be equal to $n(\underline{35})$.

Finally, given a specific isotropic geometry and receiving bandwidth, the minimum spoiling moment to provide a $2 \pi$ spin dephasing across a voxel in $T R_{1}$ amounts to:

$$
(G \delta)_{\min }=G_{\text {read }} \tau_{\text {read }}
$$

The resulting minimum spoiling moment in $T R_{2}$ is equal to $n \times(G \delta)_{\min }$, yielding a zero-order b-value difference between the readout (considering an isotropic diffusion for simplification purpose since the readout gradients vary within each repetition) and the spoiling gradients in $T R_{2}$ of about $n^{3}(\overline{43})$. Hence, the diffusion weighting contributions from the readout gradients will further be neglected.

\section{METHODS}

\section{Long and short- $T_{2}$ magnetizations behavior hypotheses}

Throughout the paper, the following hypotheses (summarized in Table 1) regarding the long and short- $T_{2}$ components are made:

- the short- $T_{2}$ component being considered to have a fully decayed transverse magnetization prior to the excitations, any contribution of diffusion weighting by spoiling 
gradients is assumed negligible as it does not affect the magnetization. Hence, its relative amplitude can be simulated using Eq. 11, and exclusively depends on $\tau_{1}, \tau_{2}$, flip angles $\alpha_{1}$ and $\alpha_{2}, T_{1}^{S}, T_{2}^{S}$ and sequence delays. Alternatively, the adiabatic inversion pulse used in IR-UTE is supposed to saturate the short- $T_{2}$ component given its assumed sub-millisecond $T_{2}$ value in simulations and experiments $(29,32)$.

- the long- $T_{2}$ component is affected by diffusion weighting through the spoiling gradients $G \delta$ and $G \delta^{\prime}$, flip angles $\alpha_{1}$ and $\alpha_{2}$, apparent diffusion coefficient $D, T_{1}^{L}, T_{2}^{L}$ and sequence delays. Similarly, relaxation during excitations is assumed negligible, and adiabatic inversion is supposed to fully invert the component's magnetization.

\section{Optimization of the Diff-UTE sequence parameters}

Once long and short- $T_{2}$ components parameters and sequence delays have been set, generating the highest contrast in the short- $T_{2}$ component consists in stretching $\tau_{1}$ to take advantage of the relaxation occurring during excitation, contracting $\tau_{2}$ to ensure a maximum excitation, and adapting $\alpha_{1}, \alpha_{2}$ and $G \delta$ to maximize the short- $T_{2}$ components signal while nulling the long- $T_{2}$ one through the states combination process.

The following is an example of algorithm for the sequence parameters choice: (i) provide expected tissues/materials properties $T_{1}^{L} / T_{2}^{L}, D$ and $T_{1}^{S} / T_{2}^{S}$; (ii) set system parameters RFphase $\Phi, T R_{2} / T R_{1}, \tau_{1}$ and $\tau_{2}$ respecting hardware limitations; (iii) set preparation pulse flip angle $\alpha_{1}$; (iv) optimize $\alpha_{2}$ to maximize the short- $T_{2}$ signal; (v) search $G \delta$ numerically to minimize the long- $T_{2}$ signal through the readout excitation pulse; (vi) if $G \delta$ is non-existent or too high for the user's hardware capacities, loop to step (iii) and modify $\alpha_{1}$ accordingly.

Even though this method allows to guarantee a theoretical short- $T_{2}$ signal maximization, converging to a suitable solution $G \delta$ may necessitate further exploration of $\alpha_{1}$ and $\alpha_{2}$. Figures S1 to S6 in Supporting Information provide additional simulations to get a better understanding of the long- $T_{2}$ signal behavior regarding the numerous parameters. 


\section{Particular considerations}

The sequence repetition time in the experiments are equal to (Fig. 11):

$$
\begin{array}{lr}
T R=\tau_{1}+T R_{1}+\tau_{2}+T R_{2} & \text { in Diff-UTE } \\
T R=\tau_{i n v}+T I+\tau_{I R}+R D & \text { in IR-UTE }
\end{array}
$$

with $\tau_{i n v}$ and $\tau_{I R}$ preparation and readout pulse durations in IR-UTE, respectively. Internal delays after preparation and readout pulses are respectively designated as $T I$ and $R D$ in the same sequence. In addition, when both methods are compared to each other, the repetition time is kept constant in both sequences so that the acquisition times are identical, and hence global signals comparable to each other.

Using the notation in Eq. [5] and Bloch equations, it can be shown that signal suppression in IR-UTE is performed by setting the TI delay to:

$$
T I=-T_{1}^{L} \ln \left(\left(1+e^{-\left(T R-\tau_{i n v}-\tau_{I R}\right) / T_{1}^{L}}\right) / 2\right)
$$

with $T_{1}^{L}$ longitudinal relaxation time of the fully inverted long- $T_{2}$ component. Additionally, due to the saturated condition of the short- $T_{2}$ magnetization after inversion (inversion efficiency $Q=0)$, the relative amplitude can be expressed as $(18)(T E=0 \mathrm{~ms})$ :

$$
S_{\mathrm{IR}-\mathrm{UTE}}^{S}=\left(1-e^{-T I / T_{1}^{S}}\right) f_{x y}^{I R}
$$

with $f_{x y}^{I R}$ the corresponding transverse mapping function of the readout pulse in IR-UTE.

Alternatively, an unprepared UTE sequence $\left(\alpha_{1}=0^{\circ}\right.$ in Diff-UTE) with an echo-subtraction technique to suppress the long- $T_{2}$ signal is considered (22) and used to quantify the expected short- $T_{2}$ signal loss through the preparation module in Diff-UTE. Using the notations of the Diff-UTE sequence, the resulting resting delay between two consecutive excitations is equal to $R D=\tau_{1}+T R_{1}+T R_{2}$. The short- $T_{2}$ signal in this sequence is therefore described by 
$(T E=0 \mathrm{~ms}):$

$$
S_{\mathrm{UTE}}^{S}=\frac{1-E_{r}}{1-E_{r} f_{z}} f_{x y}
$$

with $E_{r}=e^{-R D / T_{1}^{S}}$, and $f_{z}$ and $f_{x y}$ the corresponding mapping functions of the unique excitation pulse. From Equation [8, an optimized excitation flip angle can be evaluated similarly to the Diff-UTE short- $T_{2}$ signal optimization process, as described in (39). Finally, the ratio of both optimized signal, is expressed as:

$$
R=\frac{S_{\mathrm{Diff-UTE}}^{S}}{S_{\mathrm{UTE}}^{S}} \sqrt{2}
$$

where the $\sqrt{2}$ factor takes account of the SNR loss through the echo subtraction process in the unprepared UTE sequence to yield a theoretical long- $T_{2}$ signal suppression (21, 32).

\section{Numerical simulations}

If not otherwise stated, all numerical simulations were done using the following nominal relaxing and diffusion parameters: long- $T_{2}$ component: $D=1.00 \mu \mathrm{m}^{2} / \mathrm{ms}, T_{1}^{L} / T_{2}^{L}=$ 1000/100 ms; short- $T_{2}$ component: $T_{1}^{S} / T_{2}^{S}=300 / 0.315$ ms. In Diff-UTE, invariable parameters are intermediary delays $T R_{1} / T R_{2}=5 / 25 \mathrm{~ms}$, and readout pulse duration $\tau_{2}=0.07 \mathrm{~ms}$. The short- $T_{2}$ parameters are that of the Lego brick sample used in experiments $\left(T_{1}^{S} / T_{2}^{S}=\right.$ $300 / 0.315 \mathrm{~ms}$, assuming its $T_{2}$ value to be close to $\left.T_{2}^{*}(1,18,36)\right)$. Simulations and analyses of the long- $T_{2}$ signal were performed using the Extended Phase Graph formalism (42, 41, 44), with adapted code available at http://epg.matthias-weigel.net/ge_ssfp_epg.html.

Long- $T_{2}$ signal simulations using Diff-UTE were performed. Its signal variability along gradient spoiling moments ranging from 0.0 to $500.0 \mathrm{mT} / \mathrm{m}$.ms was evaluated for different preparation flip angles $\alpha_{1}$ ranging from $60^{\circ}$ to $90^{\circ}, \tau_{1}=1 \mathrm{~ms}$, and an optimized $\alpha_{2}$ for each value. Similarly, the short- $T_{2}$ signal is evaluated for optimized parameters given the same $\alpha_{1}$ range, and $\tau_{1}$ values from 0.5 to $3 \mathrm{~ms}$.

In order to provide insights about the robustness of the suppression in case of inaccuracies 
of the estimated relaxing and diffusion parameters, signal minima occurrences are explored through the long- $T_{2}$ relaxing and diffusion parameters. One out of three parameters is made varying about $\pm 50 \%$ of its nominal values along a constant spoiling gradient moment range from 0.0 to $300.0 \mathrm{mT} / \mathrm{m} . \mathrm{ms}$ for $\alpha_{1}=80.0^{\circ}$ and an optimized $\alpha_{2}=57.0^{\circ}$ maximizing a $T_{1}^{S} / T_{2}^{S}=300 / 0.315 \mathrm{~ms}$ species.

Specific tissues long- $T_{2}$ signal behavior were assessed in Diff-UTE. Relaxation and diffusion parameters were chosen from the literature at $3 \mathrm{~T}$ (45, 35, 46, 47): $T_{1} / T_{2}=1412 / 50 \mathrm{~ms}$ and $D=0.7 \mu \mathrm{m}^{2} / \mathrm{ms}$ for skeletal muscle, $T_{1} / T_{2}=4000 / 2000 \mathrm{~ms}$ and $D=2.2 \mu \mathrm{m}^{2} / \mathrm{ms}$ for cerebrospinal fluid, $T_{1} / T_{2}=1820 / 99 \mathrm{~ms}$ and $D=0.8 \mu \mathrm{m}^{2} / \mathrm{ms}$ for grey matter, and $T_{1} / T_{2}=1084 / 69 \mathrm{~ms}$ and $D=0.8 \mu \mathrm{m}^{2} / \mathrm{ms}$ for white matter. Preparation and excitation flip angles are $\alpha_{1} / \alpha_{2}=80 / 57^{\circ}$.

The short- $T_{2}$ signal ratio between Diff-UTE and IR-UTE is generated for the most penalizing preparation flip angle $\alpha_{1}=90^{\circ}$ and optimized $\alpha_{2}$, and for $T_{1}^{S}$ and $T_{2}^{S}$ respectively ranging from 100 to $1000 \mathrm{~ms}$ and 0.1 to $1 \mathrm{~ms}$. Alternatively, the same quantity was generated for arbitrary $\alpha_{1}$ and $\alpha_{2}$ respectively ranging from 60 to $90^{\circ}$ and 1 to $90^{\circ}$, and for $T_{1}^{S} / T_{2}^{S}=300 / 0.315 \mathrm{~ms}$. For both simulations, the IR-UTE sequence parameters are optimized to yield a long- $T_{2}$ signal cancellation having a $T_{1}^{L}$ of $1000 \mathrm{~ms}$ (i.e. $T I=11.5 \mathrm{~ms}$ ), and with $\tau_{1} / \tau_{\text {inv }} / \tau_{I R}=1 / 8 / 0.07 \mathrm{~ms}$

Finally, in order to assess the maximal signal loss induced by the preparation in DiffUTE, the $R$ ratio is computed within respective optimized conditions of $S_{\text {Diff-UTE }}^{S}$ and $S_{\mathrm{UTE}}^{S}$, and with identical excitation pulse duration and repetition time. In Diff-UTE, $\tau_{1}$ is ranging from 0.5 to $5 \mathrm{~ms}$ and $\alpha_{1}$ from 60 to $90^{\circ}$.

\section{Experimental details}

Experiments were conducted on a 7T BioSpec 70/30 USR small animal MRI system (Bruker BioSpin MRI GmbH, Ettlingen, Germany), operated with ParaVision 6.0.1. Gradient system provided a maximum amplitude of $442 \mathrm{mT} / \mathrm{m}$, and a maximum slew rate of $3660 \mathrm{~T} / \mathrm{m} / \mathrm{s}$. 
Scans were performed using a 86-mm diameter transmitter, and a mouse surface coil for reception. The native UTE-3D Bruker sequence was modified and declined into the proposed sequence (Fig. 1.a), as well as in IR-UTE (Fig. 11.b). A high-order $B_{0}$ shimming was systematically estimated prior to acquisition series. To compensate for eddy currents and gradients non-idealities (48), spokes trajectories were measured using the constructor's routine (49). Images were then reconstructed using the built-in software reconstruction pipeline.

Since the imaged phantom is considered as an homogeneous item, voxels size was chosen to be large compared to the ex vivo sample experiments in order to avoid a prohibitive total acquisition time.

\section{Phantom}

A phantom composed of an ABS plastic Lego brick immersed in a doped distilled water with $\mathrm{Ni}^{2+}, 2 \mathrm{Cl}^{-}\left(C_{\mathrm{Ni}^{2+}}=1 \mathrm{mM}\right.$; prior scans using gold-standard sequences allowed to estimate the following parameters at bore temperature $\left(\approx 20^{\circ} \mathrm{C}\right): D=1.81 \mu \mathrm{m}^{2} / \mathrm{ms}$ using a pulsed field gradient spin-echo sequence (PGSE), $T_{1}^{L}=590 \mathrm{~ms}$ using an inversion-recovery spinecho sequence (IR-SE), and $T_{2}^{L}=295 \mathrm{~ms}$ using a multi spin-echo sequence (MSE)). Relevant sequence parameters in phantom experiments were: $R F$ phase increment $\Phi_{0}=0^{\circ}$, receiver bandwidth $=150 \mathrm{kHz}$, matrix size $=96 \times 96 \times 96$, voxel size $=0.26 \mathrm{~mm}$ isotropic, number of radial lines $=28733$, dummy scans $=200$. Again, in Diff-UTE, invariable parameters are intermediary delays $T R_{1} / T R_{2}=5 / 25 \mathrm{~ms}$, and readout pulse duration $\tau_{2}=0.07 \mathrm{~ms}$.

A first estimation of the $T_{2}^{S *}$ value of the Lego brick was made using a single-pulse sequence: $\mathrm{TR}=4000 \mathrm{~ms}, 50 \mu \mathrm{s} / 90^{\circ}$ rectangular pulse, receiver bandwidth $=50 \mathrm{kHz}, 64$ averages.

The possibility of long- $T_{2}$ signal cancellation in Diff-UTE is assessed for different optimized sets of flip angles $\left\{\alpha_{1} / \alpha_{2}\right\}=\left\{90.0^{\circ} / 62.1^{\circ}, 80.0^{\circ} / 57.0^{\circ}, 70.0^{\circ} / 51.8^{\circ}, 60.0^{\circ} / 46.7^{\circ}\right\} . \tau_{1}$ and $T E$ was set to 1 and $0.02 \mathrm{~ms}$, respectively, amounting an acquisition time per scan to 14 min and 52 s. To explore the diffusion effects, 24 gradient spoiling moment values linearly 
spaced from 120.2 to $488.2 \mathrm{mT} / \mathrm{m}$.ms were used, the lowest value corresponding to a gradient spoiling moment sufficiently strong to yield an artifact-free volume.

A comparison of the short- $T_{2}$ SNR estimated in Diff-UTE and IR-UTE was conducted within long- $T_{2}$ suppression conditions. Short- $T_{2}$ signal was acquired using the same readout pulse duration $\tau_{2}=\tau_{I R}=0.07 \mathrm{~ms}$ and same $T E=0.02 \mathrm{~ms}$. For an optimal $G \delta$ value in DiffUTE, a scan was acquired using the previously proposed protocol. An 8-ms effective 1.75 kHz single-band inversion hyperbolic secant adiabatic pulse (50) was employed in IR-UTE, whose duration and bandwidth are set to be consistent to that employed in the literature which used $8.6 \mathrm{~ms}$ and $1.5 \mathrm{kHz}$ pulses $(3,29)$. TR was kept identical in both sequences in

order to equal acquisition times for each increment, while respective sequences delays obey Eq. (4) and [5. In addition, readout flip angle $\alpha_{I R}$ in IR-UTE was set to $90^{\circ}$ to ensure a maximum SNR of the short- $T_{2}$ component, and a sufficiently strong and long gradient spoiling was used to provide a dephasing moment superior to $2 \pi$ after inversion and readout. For each TR values amounting to $\{\mathrm{TR}\}=\{31.07 / 31.57 / 32.07 / 32.57 / 33.07\} \mathrm{ms}$ following an increasing $\left\{\tau_{1}\right\}=\{1.0 / 1.5 / 2.0 / 2.5 / 3.0\} \mathrm{ms}$, theoretical $\alpha_{2}$ optimal values given a constant $\alpha_{1}=80.0^{\circ}$ were used: $\left\{\alpha_{2}\right\}=\left\{57.0^{\circ} / 51.3^{\circ} / 47.4^{\circ} / 44.6^{\circ} / 42.5^{\circ}\right\}$, yielding optimal $\{G \delta\}=$ $\{159.1 / 203.3 / 229.8 / 238.7 / 247.5\} \mathrm{mT} / \mathrm{m} . \mathrm{ms}$ in Diff-UTE. In IR-UTE, given the extending TR, appropriate inversion delays were computed: $\{T I\}=\{11.5 / 11.7 / 11.9 / 12.1 / 12.3\} \mathrm{ms}$.

\section{Ex vivo sample}

A C57BL/6 adult mouse was perfused via intra-cardiac delivery of PBS followed by tissue fixation using $4 \%$ paraformaldehyde (51). All the procedures were done under deep anesthesia and in accordance to the local ethical committee rules. The head was collected and soaked into a proton-free solution (PFPE, Galden, Solvay) and scanned using both UTE sequences along with a 2D Fast Low Angle SHot (FLASH; $T R / T E=50 / 5 \mathrm{~ms}$, slice thickness $=0.5$ $\mathrm{mm}$, in-plane voxels size $=78 \times 78 \mu \mathrm{m}^{2}, 32$ averages, acquisition time $\left./ \mathrm{scan}=6 \mathrm{~min} 49 \mathrm{~s}\right)$. Prior scans (IR-SE, MSE and PGSE) allowed to estimate relaxing constants and diffusion 
characteristic in the gray matter, yielding a $T_{1}$ of $923 \pm 8.1 \mathrm{~ms}$, a $T_{2}$ of $70 \pm 2.5 \mathrm{~ms}$, and a diffusion coefficient along the spoiling direction of $0.328 \pm 0.011 \mu \mathrm{m}^{2} / \mathrm{ms}$. Approximated $T_{1}$ and $T_{2}$ values of the cortical bone at room temperature found in (52) were used for Diff-UTE parameters optimization $\left(T_{1}=120 \mathrm{~ms}, T_{2}=0.4 \mathrm{~ms}\right)$. Common parameters were $T R / T E$ $=33.07 / 0.01 \mathrm{~ms}, \mathrm{RF}$ phase increment $\Phi_{0}=0^{\circ}$, voxel size $=0.104 \mathrm{~mm}$ isotropic, matrix size $=192 \times 192 \times 192$, number of radial lines $=57527$ (undersampling factor $=2$ ) and receiver bandwidth $=200 \mathrm{kHz}$, acquisition time $/ \mathrm{scan}=31 \mathrm{~min} 42 \mathrm{~s}$. Specific parameters were $T R_{1} / T R_{2}=5 / 25 \mathrm{~ms}, \tau_{1} / \tau_{2}=3 / 0.07 \mathrm{~ms}, \alpha_{1} / \alpha_{2}=90.0 / 40.0^{\circ}$ and $G \delta=88.4 \mathrm{mT} / \mathrm{m} . \mathrm{ms}$ in Diff-UTE; 8-ms $/ 1.75 \mathrm{kHz}$ hyperbolic secant adiabatic inversion pulse, $\tau_{I R}=0.07 \mathrm{~ms}$, $\alpha_{I R}=90.0^{\circ}$ and $T I=12.4 \mathrm{~ms}$ in IR-UTE.

\section{RESULTS}

\section{Simulations}

Signal minima occurrences are depicted in blue bands on figures relating simulation results. It corresponds to an arbitrary threshold considered when the evaluated long- $T_{2}$ transverse magnetization has reached $0.05 \%$ of its value at thermal equilibrium.

As shown in Figure 2,a, signal minima are reachable given an initial tip angle $\alpha_{1}$ threshold of $76^{\circ}$ given the proposed optimization pipeline and used set of parameters. For intermediary $\alpha_{1}$ values between $79^{\circ}$ and $87^{\circ}$, two $G \delta$-ranges for suppression can be theoretically yielded, and a single range is finally obtained for $\alpha_{1}$ between $88^{\circ}$ and $90^{\circ}$. Figure 2.b illustrates the relative short- $T_{2}$ signal amplitude $S^{S}$ as a function of $\alpha_{1}$ and $\tau_{1}$. As $\tau_{1}$ increases, the relaxation-excitation effect becomes all the more important, leading to a higher short- $T_{2}$ theoretical signal. Additionally, the black dashed line indicates the domain where long- $T_{2}$ signal suppression can be achieved, and illustrates the dependency of $\alpha_{2}$ to $\tau_{1}$ through the optimization process.

Figure 3 illustrates the signal minima occurrences sensitivity about $T_{1}^{L}, T_{2}^{L}$ and $D$. The 
suppression method being based on configuration states' population modulation given the component relaxing and diffusion characteristics, the tolerance to suppression is surprisingly robust to deviations of such parameters for variations about $\pm 25 \%$. In $T_{1}$ and $T_{2}$ figures, the suppression bands join at extreme negative deviations, while the $D$ parameter seems rather robust in spite of strong deviations.

Figure 4 shows the long- $T_{2}$ signal behavior of various biological tissues. Theoretical long$T_{2}$ suppressions can rigorously be generated for every tissue, except for the skeletal muscle, most likely due to the relatively low $T_{2}$ and apparent diffusion coefficient $D$. Nonetheless, it can be considered suppressed under the threshold condition $S^{L}<0.05 \% M_{0}$ in a $G \delta$ range between 97 and $172 \mathrm{mT} / \mathrm{m} . \mathrm{ms}$.

Figure 5. a shows the ratio of short- $T_{2}$ signals generated in Diff-UTE and IR-UTE. Given the simulation parameters, the ratio reaches values below 1.0 in extreme parameters $T_{1}^{S}<$ $262 \mathrm{~ms}$ and $T_{2}^{S}>0.91 \mathrm{~ms}$, the lowest ratio being equal to 0.96 for $T_{1}^{S} / T_{2}^{S}=100 / 1 \mathrm{~ms}$. Figure 5.b shows the same ratio as a function of arbitrary $\alpha_{1}$ and $\alpha_{2}$ for $T_{1}^{S} / T_{2}^{S}=300 / 0.315 \mathrm{~ms}$. This indicates a much higher signal generation in Diff-UTE for low flip angles values, but careful attention shall be given in that a long- $T_{2}$ suppression condition is not systematically reachable in Diff-UTE for such regimes.

Figure 6 shows the ratio $R$ as a function of $\tau_{1}$ and $\alpha_{1}$. Given a constant $\alpha_{1}, R$ increases as $\tau_{1}$ does due to the increasing short- $T_{2}$ relaxation occurring during the excitation. Even though this effect allows for more short- $T_{2}$ signal generation in Diff-UTE as $\tau_{1}$ increases, the signal generated by the unprepared UTE sequence is intrinsically higher since an additional $T_{1}$ relaxation effect occurs. The highest ratio is generated for $\alpha_{1}=60^{\circ}$ and $\tau_{1}=5 \mathrm{~ms}$ $(R=0.996)$. Within the conditions in experiments (i.e. $\alpha_{1}=90^{\circ}, \tau_{1}=1 \mathrm{~ms}$ ), the estimated loss amounts to $58 \%$. 


\section{Experiments}

The acquired signal in the single pulse sequence showed a spectral line's frequency width half magnitude resulting in a $T_{2}^{S *}$ of about $315 \mu \mathrm{s}$. In IR-UTE, the appropriate TI (from Equation [6]) nulling a $T_{1}^{L}$ of $590 \mathrm{~ms}(T I=11.48 \mathrm{~ms})$ is close to that of a $T_{1}^{L}$ of $1000 \mathrm{~ms}$ component $(T I=11.53 \mathrm{~ms})$. Hence, the conditions of simulations from Figure 5.a being similar, the ratio of the short- $T_{2}$ signals generated in Diff-UTE and IR-UTE is systematically superior to 2.5 with the previously estimated $T_{2}^{S}$ for $T_{1}^{S}$ ranging from 100 to $1000 \mathrm{~ms}$. As a result and given the operating field in experiments $(7 \mathrm{~T}), T_{1}^{S}$ was further extrapolated from reported value at $105.6 \mathrm{MHz}(53)\left(T_{1} \approx 150 \mathrm{~ms}\right)$ to $300 \mathrm{~ms}$ for simulations and parameters optimization purposes.

Figure 7 shows doped-water and Lego brick signal intensities against spoiling gradient moment for four preparation flip angles $\alpha_{1}$, as well as relevant coronal views from the phantom. Maximal contrast between long- and short- $T_{2}$ components was obtained for intermediary $G \delta$ amplitudes for suppression-yielding $\alpha_{1}$ values of $80^{\circ}$ and $90^{\circ}$. Signal suppression, if any, is obviously capped to the noise level, as depicted on the graphics. Theoretical and experimental long-relaxing signals tendencies are in good agreement with each other, suggesting an appropriate description of the long- $T_{2}$ signal. While the long- $T_{2}$ signal is made varying with $G \delta$, no noticeable impact over the short- $T_{2}$ component signal at a given $\left\{\alpha_{1} ; \alpha_{2}\right\}$ pair is observed, suggesting that the proposed suppression scheme does not affect the component of interest. However, as expected, the higher the preparation flip angle $\alpha_{1}$, the lower the short- $T_{2}$ signal amplitude.

In a long- $T_{2}$ suppression condition, the effective $\mathrm{SNR}$ in the short- $T_{2}$ component is systematically higher in Diff-UTE than in IR-UTE, with respective ratios of 1.49, 1.70, 1.95, 2.03 and 2.31 for $T R$ of $31.07,31.57,32.07,32.57$ and $33.07 \mathrm{~ms}$. The ratio evolution along the increasing $T R$ shows that the suppression scheme in Diff-UTE is less penalizing than in IR-UTE regarding the short- $T_{2}$ component signal. Figure 8 illustrates the results with views of the phantom acquired at $T R$ of 31.07 and $33.07 \mathrm{~ms}$ in both sequences. 
Figure 9 shows the fixed mouse head coronal and sagittal central views in FLASH, DiffUTE and IR-UTE. Soft tissues are very well perceptible in the FLASH sequence, and no signal is visible in the head skull. On the contrary, a fairly good contrast on the cortical bone of the head skull is generated in both UTE sequences. SNR were calculated using the drawn ROI in the skull, and amounted respectively to 14.0 and 5.4 in Diff-UTE and IR-UTE, supporting simulations and previous experiments results about the short- $T_{2}$ signal generation efficiency using the proposed method.

\section{DISCUSSION}

A novel method for suppressing a long- $T_{2}$ component has been proposed. Similarly to the inversion-recovery approach, the method has proven to be efficient in suppressing contaminating signal, therefore yielding a high contrast in short- $T_{2}$ structures in an UTE acquisition sequence. Additionally, a compelling SNR in plastic polymer and cortical bone was generated through the proposed optimization process, making the approach all the more promising for further applications in imaging short- $T_{2}$ structures.

The higher SNR generated in pair with a 3D modality in the proposed method may be beneficial for various purposes. For example, sequence acceleration can be made possible through several already-established processes. Due to the expected higher short- $T_{2}$ transverse magnetization in Diff-UTE compared to the IR-UTE sequence, one can take advantage of the available short- $T_{2}$ magnetization to proceed to undersampling, or even implement a multispoke acquisition pattern within a single TR (29, 54), however at the cost of a less efficient suppression, and although limited by the required short TR to ensure a steady-state. In a clinical application, these features may consequently reduce scan time for a whole 3Dcoverage. On another hand, small voxel sizes are also responsible for a loss of SNR, as is the case in high-resolution imaging or in preclinical scanner applications, even though ultra high fields used in the latter aim to compensate for it. 
In this study, scan times may seem quite long for the ex vivo sample $(\approx 31 \mathrm{~min})$. This was due in this case i) to the relatively small voxels size yielding a rather low signal intensity and inducing SNR loss in case of a further increasing undersampling factor, and ii) to generate a distinguishable contrast in IR-UTE to be compared with the proposed method. More sensitive devices, as well as more flexible acquisition schemes (2, 55) may help taking advantage of the required short TR to reduce the acquisition scan times.

As proposed in the literature (22, 56), a second echo may be acquired after reading and refocusing the first FID. This may come in handy if ones want to suppress fat signal through a magnitude subtraction process, for example, by setting an appropriate echo time in order to provide an in-phase signal refocusing. Additional fat suppression may also be performed by appropriately including an optimized off-resonance pulse centered at $3.5 \mathrm{ppm}$ in the pulse sequence.

In ex vivo experiments, a strong global suppression and attenuation of the contaminating long- $T_{2}$ signals (white and grey matter) has been noticed. This is inherent to the short TR used in the Diff-UTE protocol, as is also the case in IR-UTE. For instance, the needed parameter - here $T I$ — does not vary much for suppressing a wide range of $T_{1}$, somehow yielding a large suppression of $T_{1}$ species, or at least a significant signal attenuation. When coupled with an efficient scheme for short- $T_{2}$ signal maximization, a substantially higher contrast to noise ratio in regions of interest is generated compared to the IR-UTE. These specific aspects may be of a great advantage in in vivo experiments as residual water signals are suppressed through an echo-subtraction technique (15, 18, 32), therefore diminishing the relative SNR in studied structures.

Another advantage of the radial 3D acquisition modality is the non-selective excitation: commonly used reshaped pulses (57) in 2D-UTE methods are sensitive to intrinsic sequence imperfections such as eddy currents inducing slice-select and readout gradients distortions (58, 59), timing errors between slice-selective gradients and RF amplifier (60, 61), and off-resonance effects that may cause slice distortions (62). These are also penalizing due 
to $T_{2}$ decay during the relatively long selective excitation (22).

No adiabatic pulse is used in Diff-UTE, but a global suppression of long relaxing signals has nonetheless been reached. This represents a great advantage in terms of RF power deposition, as well as compensating for clinical scanner limitations as the available $B_{1}$ peak is often low on such systems. For example, the pulses used in the ex vivo experiment yielded a relative SAR for the first order HS about 12.7 times higher in IR-UTE than the preparation rectangular pulse in Diff-UTE. This score was generated by using a $B_{1, \max }$ of $17 \mu \mathrm{T}$ for the HS pulse, corresponding to the minimum RF amplitude to yield an efficient inversion $(Q<-0.99(\underline{63}))$. The latter was estimated by numerically solving the Bloch equations, assuming that no relaxation occurs during the inversion. The relative SAR expressions are given in Appendix. As a result, the method is well suited for ultra-high field scanners' applications where protocols can be greatly limited by the SAR.

So far, $\alpha_{2}$ was set using the proposed optimization process where its estimation relies on the assumption of priors about $T_{1}$ and $T_{2}$ values of a single short relaxing component. However, a wide short- $T_{2}$ distribution is often found in solid-like structures, essentially due to their complexity. For example, the transverse magnetization decay of the Lego brick indicates the presence of at least two components, as shown in Figure S7. This multicomponent behavior can also be regarded as a more realistic pattern of what can be found in biological tissues (4, 64, 65, 66). Hence, assuming a single-relaxing component in such systems may result in a short- $T_{2}$ selectivity pattern since its signal will be maximized with no particular regards to other short relaxing components. As such, SNR quantification in solid-like structures may vary with the respective proton density and relaxation time constants of the various excited pools, given sets of parameters. A more complex optimization scheme for $\alpha_{2}$ optimization can be established under the condition of acknowledging the respective relaxing parameters, as well as their relative proton density fractions. An expected propagation of uncertainties regarding the experimental signal intensities may arise from such increasingly complexity. Nonetheless, a substantially higher SNR was found in our experiments when 
comparing both UTE sequences, supporting our simulations' trends.

Filtering short- $T_{2}$ and slightly short- $T_{2}$ components has also been reported in (28) through adiabatic pulses combination by taking advantage of a partial inversion effect instead of a saturation of species having $T_{2}$ above $2 \mathrm{~ms}$. While this filter is constituted by adiabatic inversion pulses in IR-UTE-like sequences, this can also be performed through steady-state and flip angles combinations in Diff-UTE. In the same manner as in IR-UTE and its adapted versions, a filtering effect of the short- $T_{2}$ components can be made through the long- $T_{2}$ suppression scheme, as well as the chosen short-relaxing compartment parameters in the maximization process, hence maximizing one component over the other. It has been shown in Figure 9 that a higher SNR can be generated with the proposed sequence compared to the IR-UTE, but no attention was given about the multicomponent behavior of the cortical bone. Further investigations may allow to discriminate appropriately the various short- $T_{2}$ pools present in such structure.

The long- $T_{2}$ suppression method was proposed in the present paper for generating contrast in sub-millisecond relaxing transverse structures. Given the advantages discussed above, an adaptation of such technique offering new kinds of selectivities (relaxation and diffusion) may represent an interesting and rapid approach for novel contrasts generation in soft tissues. Further investigations will be led accordingly.

As a prepared sequence, a loss of magnetization of the short- $T_{2}$ component is to be expected compared to an unprepared sequence with equal $T R$. An echo subtraction technique may be included in the latter for long- $T_{2}$ suppression purpose, at the cost of an inherent reduced SNR by a $\sqrt{2}$ factor $(21,32)$. Additionally, Equation 9 remains exclusively valid under the ideal condition of a fully relaxed short- $T_{2}$ component by the second echo. If not, as it might be the case for the longest $T_{2}$ component in cortical bone $\left(T_{2}^{*} \approx 1.1 \mathrm{~ms}\right.$ at $3 \mathrm{~T}$ (1)) for $T E$ about $2 \mathrm{~ms}$ for example, this will result in an increased short- $T_{2}$ signal penalization in the unprepared UTE sequence. In the same manner, the method proposed by Johnson et al. (5) takes advantage of the respective relaxing signal differences by performing 
a magnitude subtraction of an image acquired with a short pulse and a short $T E$, and an image acquired with a long pulse and a long $T E$, using the same flip angles. In spite of a doubled acquisition time, such process may yield a slightly higher short- $T_{2}$ SNR in the resulting unprepared and subtracted image since the residual short- $T_{2}$ components in the late $T E$ are less excited within the long pulse. Other issues may occur, such as additional tissue-dependent long- $T_{2}$ relaxation between echoes, diffusion weighting through readout and refocusing gradients prior to the acquisition of the second echo $(67,68)$, or even susceptibility effects (20). These irreversible effects are therefore responsible for a physical impossibility of long- $T_{2}$ suppression, all the more important when dealing with small voxels sizes and increasing operating static field.

\section{CONCLUSION}

A novel long- $T_{2}$ suppression method which employs diffusion induced weighting in a steadystate 3D-UTE sequence has been presented. Simultaneously, the sequence provides more degrees of freedom to maximize the short- $T_{2}$ signal quantity compared to the IR-UTE sequence. A good contrast was obtained with each sequence, and a compelling higher SNR was estimated in a mouse head skull in Diff-UTE compared to IR-UTE.

\section{Supporting Information}

Additional Supporting Information may be found in the online version of this article.

Fig. S1. $S^{L}$ as a function of $\alpha_{1}$ (in degrees) and $G \delta$ (in $\left.\mathrm{mT} / \mathrm{m} . \mathrm{ms}\right)$, for $T_{2}^{L}=25 / 50 / 100 / 1000$ ms (columns), and $\alpha_{2}=15 / 30 / 45 / 60 / 75 / 90^{\circ}$ (rows). Other simulation parameters: $T_{1}^{L}=$ $1000 \mathrm{~ms}, D=0.3 \mu \mathrm{m}^{2} / \mathrm{ms}$ and $T R_{1} / T R_{2}=5 / 25 \mathrm{~ms}$. Signal minima occurences $\left(S^{L}<\right.$ $\left.0.05 \% M_{0}\right)$ are depicted in the blue bands.

Fig. S2. $S^{L}$ as a function of $\alpha_{1}$ (in degrees) and $G \delta$ (in $\mathrm{mT} / \mathrm{m} . \mathrm{ms}$ ), for $T_{2}^{L}=25 / 50 / 100 / 1000$ ms (columns), and $\alpha_{2}=15 / 30 / 45 / 60 / 75 / 90^{\circ}$ (rows). Other simulation parameters: $T_{1}^{L}=$ 
$1000 \mathrm{~ms}, D=0.8 \mu \mathrm{m}^{2} / \mathrm{ms}$ and $T R_{1} / T R_{2}=5 / 25 \mathrm{~ms}$. Signal minima occurences $\left(S^{L}<\right.$ $\left.0.05 \% M_{0}\right)$ are depicted in the blue bands.

Fig. S3. $S^{L}$ as a function of $\alpha_{1}$ (in degrees) and $G \delta$ (in $\mathrm{mT} / \mathrm{m} . \mathrm{ms}$ ), for $T_{2}^{L}=25 / 50 / 100 / 1000$ ms (columns), and $\alpha_{2}=15 / 30 / 45 / 60 / 75 / 90^{\circ}$ (rows). Other simulation parameters: $T_{1}^{L}=$ $1000 \mathrm{~ms}, D=2.0 \mu \mathrm{m}^{2} / \mathrm{ms}$ and $T R_{1} / T R_{2}=5 / 25 \mathrm{~ms}$. Signal minima occurences $\left(S^{L}<\right.$ $\left.0.05 \% M_{0}\right)$ are depicted in the blue bands.

Fig. S4. $S^{L}$ as a function of $\alpha_{1}$ (in degrees) and $G \delta$ (in $\mathrm{mT} / \mathrm{m} . \mathrm{ms}$ ), for $T_{2}^{L}=25 / 50 / 100 / 1000$ ms (columns), and $\alpha_{2}=15 / 30 / 45 / 60 / 75 / 90^{\circ}$ (rows). Other simulation parameters: $T_{1}^{L}=$ $1400 \mathrm{~ms}, D=0.3 \mu \mathrm{m}^{2} / \mathrm{ms}$ and $T R_{1} / T R_{2}=5 / 25 \mathrm{~ms}$. Signal minima occurences $\left(S^{L}<\right.$ $\left.0.05 \% M_{0}\right)$ are depicted in the blue bands.

Fig. S5. $S^{L}$ as a function of $\alpha_{1}$ (in degrees) and $G \delta$ (in $\left.\mathrm{mT} / \mathrm{m} . \mathrm{ms}\right)$, for $T_{2}^{L}=25 / 50 / 100 / 1000$ ms (columns), and $\alpha_{2}=15 / 30 / 45 / 60 / 75 / 90^{\circ}$ (rows). Other simulation parameters: $T_{1}^{L}=$ $1400 \mathrm{~ms}, D=0.8 \mu \mathrm{m}^{2} / \mathrm{ms}$ and $T R_{1} / T R_{2}=5 / 25 \mathrm{~ms}$. Signal minima occurences $\left(S^{L}<\right.$ $\left.0.05 \% M_{0}\right)$ are depicted in the blue bands.

Fig. S6. $S^{L}$ as a function of $\alpha_{1}$ (in degrees) and $G \delta$ (in $\mathrm{mT} / \mathrm{m} . \mathrm{ms}$ ), for $T_{2}^{L}=25 / 50 / 100 / 1000$ ms (columns), and $\alpha_{2}=15 / 30 / 45 / 60 / 75 / 90^{\circ}$ (rows). Other simulation parameters: $T_{1}^{L}=$ $1400 \mathrm{~ms}, D=2.0 \mu \mathrm{m}^{2} / \mathrm{ms}$ and $T R_{1} / T R_{2}=5 / 25 \mathrm{~ms}$. Signal minima occurences $\left(S^{L}<\right.$ $\left.0.05 \% M_{0}\right)$ are depicted in the blue bands.

Fig. S7. Fittings of the Lego brick experimental signal (circles). Bi-component fitting is depicted in dashed line, mono-component in dotted line, and noise level in crosses. DiffUTE parameters were: $\mathrm{TR}=31.07 \mathrm{~ms}\left(T R_{1} / T R_{2}=5 / 25 \mathrm{~ms}, \tau_{1} / \tau_{2}=1 / 0.07 \mathrm{~ms}\right), \alpha_{1} / \alpha_{2}=$ $80.0 / 57.0^{\circ}, G \delta=159.12 \mathrm{mT} / \mathrm{m} . \mathrm{ms}$, and $T E=0.02,0.03,0.05,0.10,0.15,0.25,0.40,1.10,1.80$ ms. Bi-component estimation yielded $T_{2}^{* S, 1}=0.18 \pm 0.05 \mathrm{~ms}\left(F^{S, 1}=57.6 \pm 9.5 \%\right)$, and $T_{2}^{* S, 2}=1.63 \pm 0.52 \mathrm{~ms}\left(F^{S, 2}=42.4 \pm 10.4 \%\right)\left(R_{a d j}^{2}=0.99\right)$, and mono-component $T_{2}^{*}=$ $0.52 \pm 0.09 \mathrm{~ms}\left(R_{a d j}^{2}=0.94\right)$. 


\section{ACKNOWLEDGEMENTS}

The authors thank Dr. Arnaud Duchon for providing the fixed mouse head used in experiments, as well as Drs. Laura Harsan and Ericky C. A. Araujo for the careful manuscript reviews.

\section{APPENDIX}

\section{Short- $\mathrm{T}_{2}$ signal derivation}

The signal of interest $S^{S}$ is acquired after the excitation pulse $\left\{\alpha_{2} / \tau_{2}\right\}$. Assuming a complete spoiling before each excitation (i.e. $T_{2} \ll\left\{T R_{1} ; T R_{2}\right\}$ ), one can track the short- $T_{2}$ longitudinal magnetization from one repetition to another in a steady-state condition using the Bloch equations :

$$
\left\{\begin{array}{l}
M_{z}^{S}\left(\tau_{2}\right)=M_{z}^{S}\left(T R_{1}\right) \times f_{z_{2}} \\
M_{z}^{S}\left(T R_{2}\right)=M_{0}-\left(M_{0}-M_{z}^{S}\left(\tau_{2}\right)\right) E_{w} \\
M_{z}^{S}\left(\tau_{1}\right)=M_{z}^{S}\left(T R_{2}\right) \times f_{z_{1}} \\
M_{z}^{S}\left(T R_{1}\right)=M_{0}-\left(M_{0}-M_{z}^{S}\left(\tau_{1}\right)\right) E_{s}
\end{array}\right.
$$

noting $M_{z}^{S}\left(t_{i}\right)$ the longitudinal magnetization after the delay $t_{i}$, and with $E_{s}=e^{-T R_{1} / T_{1}^{S}}$, $E_{w}=e^{-T R_{2} / T_{1}^{S}}\left(T R_{1}\right.$ and $T R_{2}$ referring to the internal delays after excitations), $f_{z_{i}}=$

$e^{-w_{i}}\left[\cos \left(\gamma_{i}\right)+w_{i} \operatorname{sinc}\left(\gamma_{i}\right)\right]$ (with $\gamma_{i}=\sqrt{\alpha_{i}^{2}-w_{i}^{2}}$ and $\left.w_{i}=\tau_{i} / 2 T_{2}^{S}\right), \alpha_{i}$ and $\tau_{i}$ being the respective flip angles and pulse durations. It therefore yields :

$$
M_{z}^{S}\left(T R_{1}\right)=M_{0} \frac{\left(1-E_{s}\right)+E_{s}\left(1-E_{w}\right) f_{z_{1}}}{1-f_{z_{1}} f_{z_{2}} E_{s} E_{w}}
$$

providing the normalized transverse magnetization after the excitation pulse in equation [1]. 


\section{SAR expressions}

With no particular regards about the duty cycle, the relative SAR $\Gamma$ of the pulses used in experiments can be expressed as (69, 70) :

$$
\begin{array}{lr}
\Gamma_{H S} \propto \frac{B_{1, \max }^{2} \tau_{I R}}{\beta} & \text { for a first order HS pulse }, \\
\Gamma_{B P} \propto B_{1, \max }^{2} \tau_{1} & \text { for a rectangular pulse },
\end{array}
$$

with $B_{1, \max }$ the respective pulse maximum amplitude, and $\beta \approx 5.3$ truncation factor of the HS pulse.

\section{References}

1. Du J, Carl M, Bydder M, Takahashi A, Chung CB, Bydder GM. Qualitative and quantitative ultrashort echo time (UTE) imaging of cortical bone. Journal of Magnetic Resonance 2010;207:304-311.

2. Chen J, Chang EY, Carl M, Ma Y, Shao H, Chen B, Wu Z, Du J. Measurement of bound and pore water T1 relaxation times in cortical bone using three-dimensional ultrashort echo time cones sequences. Magnetic Resonance in Medicine 2017;77:2136-2145.

3. Chen J, Carl M, Ma Y, Shao H, Lu X, Chen B, Chang EY, Wu Z, Du J. Fast volumetric imaging of bound and pore water in cortical bone using three-dimensional ultrashort-TE (UTE) and inversion recovery UTE sequences. NMR in Biomedicine 2016;29:1373-1380.

4. Horch RA, Nyman JS, Gochberg DF, Dortch RD, Does MD. Characterization of $1 \mathrm{H}$ NMR signal in human cortical bone for magnetic resonance imaging. Magnetic Resonance in Medicine 2010;64:680-687.

5. Johnson EM, Vyas U, Ghanouni P, Pauly KB, Pauly JM. Improved cortical bone specificity in UTE MR Imaging. Magnetic Resonance in Medicine 2017;77:684-695. 
6. Wiesinger F, Sacolick LI, Menini A, Kaushik SS, Ahn S, VeitHaibach P, Delso G, Shanbhag DD. Zero TE MR bone imaging in the head. Magnetic Resonance in Medicine 2015;114:107-114.

7. Weiger M, Wu M, Wurnig MC, Kenkel D, Boss A, Andreisek G, Pruessmann KP. ZTE imaging with long- T 2 suppression. NMR in Biomedicine 2015;28:247-254.

8. Nebelung S, Tingart M, Pufe T, Kuhl C, Jahr H, Truhn D. Ex vivo quantitative multiparametric MRI mapping of human meniscus degeneration. Skeletal Radiology 2016;45:1649-1660.

9. Williams A, Qian Y, Golla S, Chu CR. UTE-T2* mapping detects sub-clinical meniscus injury after anterior cruciate ligament tear. Osteoarthritis and Cartilage 2012;20:486494.

10. Chang EY, Du J, Iwasaki K, Biswas R, Statum S, He Q, Bae WC, Chung CB. Singleand Bi-component $\mathrm{T} 2 *$ analysis of tendon before and during tensile loading, using UTE sequences. Journal of Magnetic Resonance Imaging 2015;42:114-120.

11. Ma YJ, Shao H, Du J, Chang EY. Ultrashort echo time magnetization transfer (UTEMT) imaging and modeling: magic angle independent biomarkers of tissue properties. NMR in Biomedicine 2016;29:1546-1552.

12. Du J, Pak BC, Znamirowski R, Statum S, Takahashi A, Chung CB, Bydder GM. Magic angle effect in magnetic resonance imaging of the Achilles tendon and enthesis. Magnetic Resonance Imaging 2009;27:557-564.

13. Wright P, Jellus V, McGonagle D, Robson M, Ridgeway J, Hodgson R. Comparison of two ultrashort echo time sequences for the quantification of $\mathrm{T} 1$ within phantom and human Achilles tendon at 3 T. Magnetic Resonance in Medicine 2012;68:1279-1284. 
14. Johnson KM, Fain SB, Schiebler ML, Nagle S. Optimized 3D ultrashort echo time pulmonary MRI. Magnetic Resonance in Medicine 2013;70:1241-1250.

15. Tibiletti M, Bianchi A, Stiller D, Rasche V. Pulmonary perfusion quantification with flow-sensitive inversion recovery (FAIR) UTE MRI in small animal imaging. NMR in Biomedicine 2016;29:1791-1799.

16. Gai ND, Malayeri AA, Bluemke DA. Three-dimensional T1 and T2* mapping of human lung parenchyma using interleaved saturation recovery with dual echo ultrashort echo time imaging (ITSR-DUTE). Journal of Magnetic Resonance Imaging 2017;45:10971104.

17. Du J, Ma G, Li S, Carl M, Szeverenyi NM, VandenBerg S, CoreyBloom J, Bydder GM. Ultrashort echo time (UTE) magnetic resonance imaging of the short T2 components in white matter of the brain using a clinical 3T scanner. NeuroImage 2014;87:32-41.

18. Du J, Sheth V, He Q, Carl M, Chen J, CoreyBloom J, Bydder GM. Measurement of T1 of the Ultrashort T2* Components in White Matter of the Brain at 3T. PLoS ONE 2014;9:e103296.

19. Sheth V, Shao H, Chen J, Vandenberg S, CoreyBloom J, Bydder GM, Du J. Magnetic resonance imaging of myelin using ultrashort Echo time (UTE) pulse sequences: Phantom, specimen, volunteer and multiple sclerosis patient studies. NeuroImage 2016;136:37-44.

20. Du J, Bydder M, Takahashi AM, Carl M, Chung CB, Bydder GM. Short T2 contrast with three-dimensional ultrashort echo time imaging. Magnetic Resonance Imaging 2011;29:470-482.

21. Larson PEZ, Gurney PT, Nayak K, Gold GE, Pauly JM, Nishimura DG. Designing long-T2 suppression pulses for ultrashort echo time imaging. Magnetic Resonance in Medicine 2006;56:94-103. 
22. Robson MD, Gatehouse PD, Bydder M, Bydder GM. Magnetic resonance: an introduction to ultrashort TE (UTE) imaging. Journal of computer assisted tomography $2003 ; 27: 825-46$.

23. Lee YH, Kim S, Song HT, Kim I, Suh JS. Weighted subtraction in 3D ultrashort echo time (UTE) imaging for visualization of short T2 tissues of the knee. Acta Radiologica 2014;55:454-461.

24. Deligianni X, Bär P, Scheffler K, Trattnig S, Bieri O. Water-selective excitation of short T2 species with binomial pulses. Magnetic Resonance in Medicine 2014;72:800-805.

25. Tyler DJ, Robson MD, Henkelman RM, Young IR, Bydder GM. Magnetic resonance imaging with ultrashort TE (UTE) PULSE sequences: Technical considerations. Journal of Magnetic Resonance Imaging 2007;25:279-289.

26. Du J, Takahashi AM, Bae WC, Chung CB, Bydder GM. Dual inversion recovery, ultrashort echo time (DIR UTE) imaging: Creating high contrast for short-T2 species. Magnetic Resonance in Medicine 2010;63:447-455.

27. Larson PEZ, Conolly SM, Pauly JM, Nishimura DG. Using adiabatic inversion pulses for long-T2 suppression in ultrashort echo time (UTE) imaging. Magnetic Resonance in Medicine 2007;58:952-961.

28. Horch RA, Gochberg DF, Nyman JS, Does MD. Clinically compatible MRI strategies for discriminating bound and pore water in cortical bone. Magnetic Resonance in Medicine $2012 ; 68: 1774-1784$.

29. Carl M, Bydder GM, Du J. UTE imaging with simultaneous water and fat signal suppression using a time-efficient multispoke inversion recovery pulse sequence. Magnetic Resonance in Medicine 2016;76:577-582. 
30. He Q, Ma Y, Fan S, Shao H, Sheth V, Bydder GM, Du J. Direct magnitude and phase imaging of myelin using ultrashort echo time (UTE) pulse sequences: A feasibility study. Magnetic Resonance Imaging 2017;39:194-199.

31. Gai ND, Malayeri AA, Bluemke DA. Long T2 suppression in native lung 3-D imaging using k-space reordered inversion recovery dual-echo ultrashort echo time MRI. Magnetic Resonance Materials in Physics, Biology and Medicine 2017;pp. 1-9.

32. Li C, Magland JF, Rad HS, Song HK, Wehrli FW. Comparison of optimized soft-tissue suppression schemes for ultrashort echo time MRI. Magnetic Resonance in Medicine 2012;68:680-689.

33. Soustelle L, Lamy J, Loureiro de Sousa P, Rousseau F, Armspach J. Long-T2 suppression based on saturation and diffusion in a steady-state 3D-UTE sequence. in Proceedings of the 25th Annual Meeting of ISMRM, Honolulu, Hawaii, USA, 2017. p. 4031.

34. Yarnykh VL. Actual flip-angle imaging in the pulsed steady state: A method for rapid three-dimensional mapping of the transmitted radiofrequency field. Magnetic Resonance in Medicine 2007;57:192-200.

35. Nehrke K. On the steady-state properties of actual flip angle imaging (AFI). Magnetic Resonance in Medicine 2009;61:84-92.

36. Sussman MS, Pauly JM, Wright GA. Design of practical T2-selective RF excitation (TELEX) pulses. Magnetic Resonance in Medicine 1998;40:890-899.

37. Torrey HC. Transient Nutations in Nuclear Magnetic Resonance. Physical Review 1949;76:1059-1068.

38. Hu BS, Conolly SM, Wright Ga, Nishimura DG, Macovski A. Pulsed saturation transfer contrast. Magnetic Resonance in Medicine 1992;26:231-240. 
39. Carl M, Bydder M, Du J, Takahashi A, Han E. Optimization of RF excitation to maximize signal and T2 contrast of tissues with rapid transverse relaxation. Magnetic Resonance in Medicine 2010;64:481-90.

40. Gudbjartsson H, Patz S. Simultaneous calculation of flow and diffusion sensitivity in steady- state free precession imaging. Magnetic Resonance in Medicine 1995;34:567-579.

41. Weigel M, Schwenk S, Kiselev V, Scheffler K, Hennig J. Extended phase graphs with anisotropic diffusion. Journal of Magnetic Resonance 2010;205:276-285.

42. Weigel M. Extended phase graphs: Dephasing, RF pulses, and echoes - pure and simple. Journal of Magnetic Resonance Imaging 2015;41:266-295.

43. Mattiello J, Basser PJ, Bihan DL. Matrix in Diffusion Tensor Echo-Planar Imaging. Methods 1996;37:292-300.

44. Hennig J. Echoes - how to generate, recognize, use or avoid them in MR-imaging sequences. Part I: Fundamental and not so fundamental properties of spin echoes. Concepts in Magnetic Resonance 1991;3:125-143.

45. Stanisz GJ, Odrobina EE, Pun J, Escaravage M, Graham SJ, Bronskill MJ, Henkelman RM. T1, T2 relaxation and magnetization transfer in tissue at 3T. Magnetic Resonance in Medicine 2005;54:507-512.

46. Rooney WD, Johnson G, Li X, Cohen ER, Kim SG, Ugurbil K, Springer CS. Magnetic field and tissue dependencies of human brain longitudinal1H2O relaxation in vivo. Magnetic Resonance in Medicine 2007;57:308-318.

47. Bojorquez JZ, Bricq S, Acquitter C, Brunotte F, Walker PM, Lalande A. What are normal relaxation times of tissues at 3 T? Magnetic Resonance Imaging 2017;35:69-80.

48. Brodsky EK, Klaers JL, Samsonov AA, Kijowski R, Block WF. Rapid measurement 
and correction of phase errors from B0 eddy currents: Impact on image quality for non-cartesian imaging. Magnetic Resonance in Medicine 2013;69:509-515.

49. Duyn JH, Yang Y, Frank JA, van der Veen JW. Simple correction method for k-space trajectory deviations in MRI. Journal of magnetic resonance (San Diego, Calif. : 1997) 1998;132:150-3.

50. Silver M, Joseph R, Hoult D. Highly selective and $\pi$ pulse generation. Journal of Magnetic Resonance 1984;59:347-351.

51. Cahill LS, Laliberté CL, Ellegood J, Spring S, Gleave Ja, van Eede MC, Lerch JP, Henkelman RM. Preparation of fixed mouse brains for MRI. NeuroImage 2012;60:933939.

52. Han M, Rieke V, Scott SJ, Ozhinsky E, Salgaonkar VA, Jones PD, Larson PEZ, Diederich CJ, Krug R. Quantifying temperature-dependent T1 changes in cortical bone using ultrashort echo-time MRI. Magnetic Resonance in Medicine 2015;74:1548-1555.

53. Codd S, Mallett M, Halse M, Strange J, Vennart W, Doorn T. A Three-Dimensional NMR Imaging Scheme Utilizing Doubly Resonant Gradient Coils. Journal of Magnetic Resonance, Series B 1996;113:214-221.

54. Li C, Magland JF, Zhao X, Seifert AC, Wehrli FW. Selective in vivo bone imaging with long-T2 suppressed PETRA MRI. Magnetic Resonance in Medicine 2017;77:989-997.

55. Gurney PT, Hargreaves Ba, Nishimura DG. Design and analysis of a practical 3D cones trajectory. Magnetic Resonance in Medicine 2006;55:575-582.

56. Waldman a, Rees JH, Brock CS, Robson MD, Gatehouse PD, Bydder GM. MRI of the brain with ultra-short echo-time pulse sequences. Neuroradiology 2003;45:887-92.

57. Pauly J, Conolly SM. Slice-selective excitation for very short T2 species. in Proceedings of the 8th Annual Meeting of SMRM, Amsterdam, The Netherlands, 1989. p. 28. 
58. Harkins KD, Horch RA, Does MD. Simple and robust saturation-based slice selection for ultrashort echo time MRI. Magnetic Resonance in Medicine 2015;73:2204-2211.

59. Josan S, Pauly JM, Daniel BL, Pauly KB. Double half RF pulses for reduced sensitivity to eddy currents in UTE imaging. Magnetic resonance in medicine : official journal of the Society of Magnetic Resonance in Medicine / Society of Magnetic Resonance in Medicine 2009;61:1083-9.

60. Soustelle L, Loureiro de Sousa P, Koehler S, Po C, Rousseau F, Armspach J. MinimumTime VERSE Pulse Correction for Slice Selectivity Improvement in 2D-UTE Imaging. in Proceedings of the 24th Annual Meeting of ISMRM, Singapore, Singapore, 2016. p. 1888.

61. Harkins KD, Manhard K, Grissom WA, Does MD. Automatic Gradient Predistortion Applied to Clinical 2D-UTE. in Proceedings of the 24th Annual Meeting of ISMRM, Singapore, Singapore, 2016. p. 4289.

62. Pauly JM. Selective Excitation for Ultrashort Echo Time Imaging. in Encyclopedia of Magnetic Resonance, Vol. 1, pp. 1-7. John Wiley \& Sons, Ltd, Chichester, UK, 2012.

63. Garwood M, DelaBarre L. The return of the frequency sweep: designing adiabatic pulses for contemporary NMR. Journal of magnetic resonance (San Diego, Calif. : 1997) 2001;153:155-177.

64. Horch RA, Gore JC, Does MD. Origins of the ultrashort-T2 1H NMR signals in myelinated nerve: A direct measure of myelin content? Magnetic Resonance in Medicine $2011 ; 66: 24-31$.

65. Wilhelm MJ, Ong HH, Wehrli SL, Li C, Tsai PH, Hackney DB, Wehrli FW. Direct magnetic resonance detection of myelin and prospects for quantitative imaging of myelin density. Proceedings of the National Academy of Sciences 2012;109:9605-9610. 
66. Rad HS, Lam SCB, Magland JF, Ong H, Li C, Song HK, Love J, Wehrli FW. Quantifying cortical bone water in vivo by three-dimensional ultra-short echo-time MRI. NMR in Biomedicine 2011;24:855-864.

67. Chaudhari AS, Sveinsson B, Moran CJ, McWalter EJ, Johnson EM, Zhang T, Gold GE, Hargreaves BA. Imaging and T2 relaxometry of short-T2 connective tissues in the knee using ultrashort echo-time double-echo steady-state (UTEDESS). Magnetic Resonance in Medicine 2017;00.

68. Bieri O, Ganter C, Scheffler K. On the fluid-tissue contrast behavior of high-resolution steady-state sequences. Magnetic Resonance in Medicine 2012;68:1586-1592.

69. Idiyatullin D, Corum C, Moeller S, Garwood M. Gapped pulses for frequency-swept MRI. Journal of Magnetic Resonance 2008;193:267-273.

70. Bernstein MA, King KE, Zhou XJ, Fong W. Handbook of MRI Pulse Sequences. Medical Physics 2005;32:1452. 


\section{List of Figures}

1 Diff-UTE (a) and IR-UTE (b) pulse sequences. The gradients inducing diffusion weighting in Diff-UTE correspond respectively to the short one after the preparation pulse (relative area $G \delta$ ), and to the long one after the readout gradients refocusing

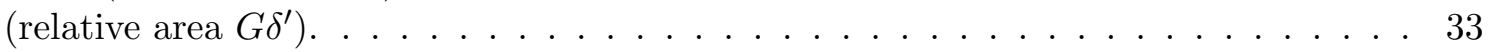

$2 \quad$ Simulation of long- $T_{2}$ signals along preparation flip angle $\alpha_{1}$ and spoiling gradient moment $G \delta$ for $\tau_{1}=1 \mathrm{~ms}(\mathrm{a})$, and short- $T_{2}$ signals along $\alpha_{1}$ and $\tau_{1}$ (b). In both cases, $\alpha_{2}$ was set to its optimized value given $\alpha_{1}$ and $\tau_{1}$. The dashed line in (b) indicates the lowest $\alpha_{1}$ limit value as a function of $\tau_{1}$ for the proposed optimization scheme to yield a long- $T_{2}$ suppression $\left(S^{L}<0.05 \% M_{0}\right)$, and the white crosses in (a) and (b) relates this aspect for $\tau_{1}=1 \mathrm{~ms}$. Simulation parameters are $T_{1}^{L} / T_{2}^{L}=1000 / 100 \mathrm{~ms}$, $T_{1}^{S} / T_{2}^{S}=300 / 0.315 \mathrm{~ms}, D=1.00 \mu \mathrm{m}^{2} / \mathrm{ms}, \tau_{2}=0.07 \mathrm{~ms}, T R_{1} / T R_{2}=5 / 25 \mathrm{~ms}$. Signal minima occurrences are depicted in the blue bands. $\ldots \ldots \ldots 35$

3 Simulated long- $T_{2}$ signals as functions of spoiling gradient moment and $T_{1}^{L}((\mathrm{a})$ nominal $\left.T_{1}^{L}=1000 \mathrm{~ms}\right), T_{2}^{L}\left((\mathrm{~b})-\right.$ nominal $\left.T_{2}^{L}=100 \mathrm{~ms}\right)$ and $D((\mathrm{c})-$ nominal $\left.D=1.00 \mu \mathrm{m}^{2} / \mathrm{ms}\right)$ deviations. Simulation parameters: $T_{1}^{S} / T_{2}^{S}=300 / 0.315 \mathrm{~ms}$, $\tau_{1} / \tau_{2}=1 / 0.07 \mathrm{~ms}, \alpha_{1} / \alpha_{2}=80 / 57.0^{\circ}$ and $T R_{1} / T R_{2}=5 / 25 \mathrm{~ms}$. Signal minima occurrences $\left(S^{L}<0.05 \% M_{0}\right)$ are depicted in the blue bands. $\ldots \ldots . . . . .36$

4 Simulated long- $T_{2}$ signals as functions of spoiling gradient moment for biological tissues: skeletal muscle (MSK, dashed line, $T_{1} / T_{2}=1412 / 50 \mathrm{~ms}, D=0.7 \mu \mathrm{m}^{2} / \mathrm{ms}$ ), cerebrospinal fluid (CSF, solid line, $T_{1} / T_{2}=4000 / 2000 \mathrm{~ms}, D=2.2 \mu \mathrm{m}^{2} / \mathrm{ms}$ ), grey matter (GM, dotted line, $T_{1} / T_{2}=1820 / 99 \mathrm{~ms}, D=0.8 \mu \mathrm{m}^{2} / \mathrm{ms}$ ) and white matter (WM, dashed-dotted line, $T_{1} / T_{2}=1084 / 69 \mathrm{~ms}, D=0.8 \mu \mathrm{m}^{2} / \mathrm{ms}$ ). Other simulation parameters: $T_{1}^{S} / T_{2}^{S}=300 / 0.315 \mathrm{~ms}, \tau_{1} / \tau_{2}=1 / 0.07 \mathrm{~ms}, \alpha_{1} / \alpha_{2}=80 / 57.0^{\circ}$ and $T R_{1} / T R_{2}=5 / 25 \mathrm{~ms}$. The horizontal blue line indicates the suppression threshold

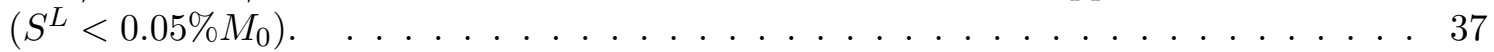

$5 \quad$ Simulated short- $T_{2}$ signal ratio as a function of $T_{1}^{S}$ and $T_{2}^{S}$ for the most penalizing case of a preparation flip angle $\alpha_{1}=90^{\circ}$ and optimized $\alpha_{2}$ (a), and as a function of $\alpha_{1}$ and $\alpha_{2}$ for $T_{1}^{S} / T_{2}^{S}=300 / 0.315 \mathrm{~ms}$ (b). The black dashed line in (a) corresponds to the estimated $T_{2}^{S}=315 \mu$ s value in the Lego brick. The black solid line in (b) corresponds to the optimized $\alpha_{2}$ maximizing Eq. [1]. Other simulation parameters are $\tau_{1} / \tau_{2}=1 / 0.07 \mathrm{~ms}$, and $T R_{1} / T R_{2}=5 / 25 \mathrm{~ms}$ in Diff-UTE, $\tau_{\text {inv }} / \tau_{I R}=8 / 0.07 \mathrm{~ms}$, and $T I / R D=11.5 / 11.5 \mathrm{~ms}$ in IR-UTE (optimized delays to suppress a component

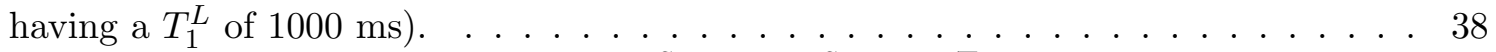

$6 \quad$ Simulated short- $T_{2}$ signals ratio $R=\left(S_{\text {Diff-UTE }}^{S} / S_{\text {UTE }}^{S}\right) \times \sqrt{2}$ as a function of $\tau_{1}$ and $\alpha_{1}$ for respective optimized $\alpha_{2}$ flip angle. Simulation parameters are $T_{1}^{S} / T_{2}^{S}=300 / 0.315$ $\mathrm{ms}, \tau_{2}=0.07 \mathrm{~ms}$ and $T R_{1} / T R_{2}=5 / 25 \mathrm{~ms} . \quad \ldots \ldots \ldots \ldots$ 
$7 \quad$ Effect of the Diff-UTE suppression scheme for four preparation flip angle $\alpha_{1}$ values: $\alpha_{1}=60^{\circ}$ (a), $\alpha_{1}=70^{\circ}$ (b), $\alpha_{1}=80^{\circ}$ (c) and $\alpha_{1}=90^{\circ}$ (d) against spoiling gradient moment $G \delta$. Doped-water (circle) and short- $T_{2}$ component (cross) correspond to the mean signal intensities estimated respectively in ROIs A and B drawn in the view (e). The solid line describes the simulated water signal amplitude given the experimental parameters. The noise ROI was drawn away in an artifact-free area (not shown), and its evolution is depicted in bullets. All experimental signals on plots of the different scenarios are normalized to the water signal's intensity corresponding to $\alpha_{1}=60^{\circ}$ and $G \delta=488 \mathrm{mT} / \mathrm{m} . \mathrm{ms}$, the latter being considered to be fully spoiled (the signal evolution being very slow afterwards). Coronal views (e-h) were chosen given relevant spoiling gradient moment values (dashed vertical lines on corresponding plots $\{\mathrm{a}, \mathrm{e}\},\{\mathrm{b}, \mathrm{f}\},\{\mathrm{c}, \mathrm{g}\},\{\mathrm{d}, \mathrm{h}\})$ where the contrast of the short- $T_{2}$ component is either negative (e), null (f) or positive $(\mathrm{g}, \mathrm{h})$, consecutively to the long relaxing component

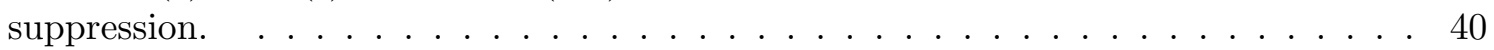

8 Coronal slices acquired in Diff-UTE for $\tau_{1}$ of 1.0 (a) and $3.0 \mathrm{~ms}$ (b) in Diff-UTE, and for $T I$ of 11.5 (c) and $12.3 \mathrm{~ms}(\mathrm{~d})$ in IR-UTE. The same contrast window levels is used in each view. $T R$ is kept constant in both sequences in each column $\{\mathrm{a}, \mathrm{c}\}$ and $\{\mathrm{b}, \mathrm{d}\}$. Experimental SNR ratios of the short- $T_{2}$ component generated between DiffUTE and IR-UTE amount to 1.49 for $T R=31.07 \mathrm{~ms}$, and 2.31 for $T R=33.07 \mathrm{~ms}$.

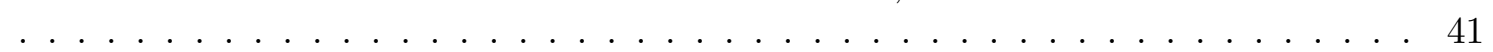

9 Coronal (top row) and sagittal (bottom row) views of the fixed mouse head in FLASH (a), Diff-UTE (b) and IR-UTE (c). The same dynamic range was used in all four UTE images. The bone ROI used for SNR analysis between Diff-UTE and IR-UTE is drawn in red on the sagittal view of the Diff-UTE column (b). The noise ROI (not shown) was drawn far from the head in an artifact-free area. . . . . . . . . . . . . . 42

\section{List of Tables}

$1 \quad$ Summary of the components' relevant parameters influencing their magnetization. Symbols in parenthesis, if any, indicate the tendencies of the corresponding parameter that would maximize the short- $T_{2}$ component signal in Diff-UTE. . . . . . . . . . . . 34 

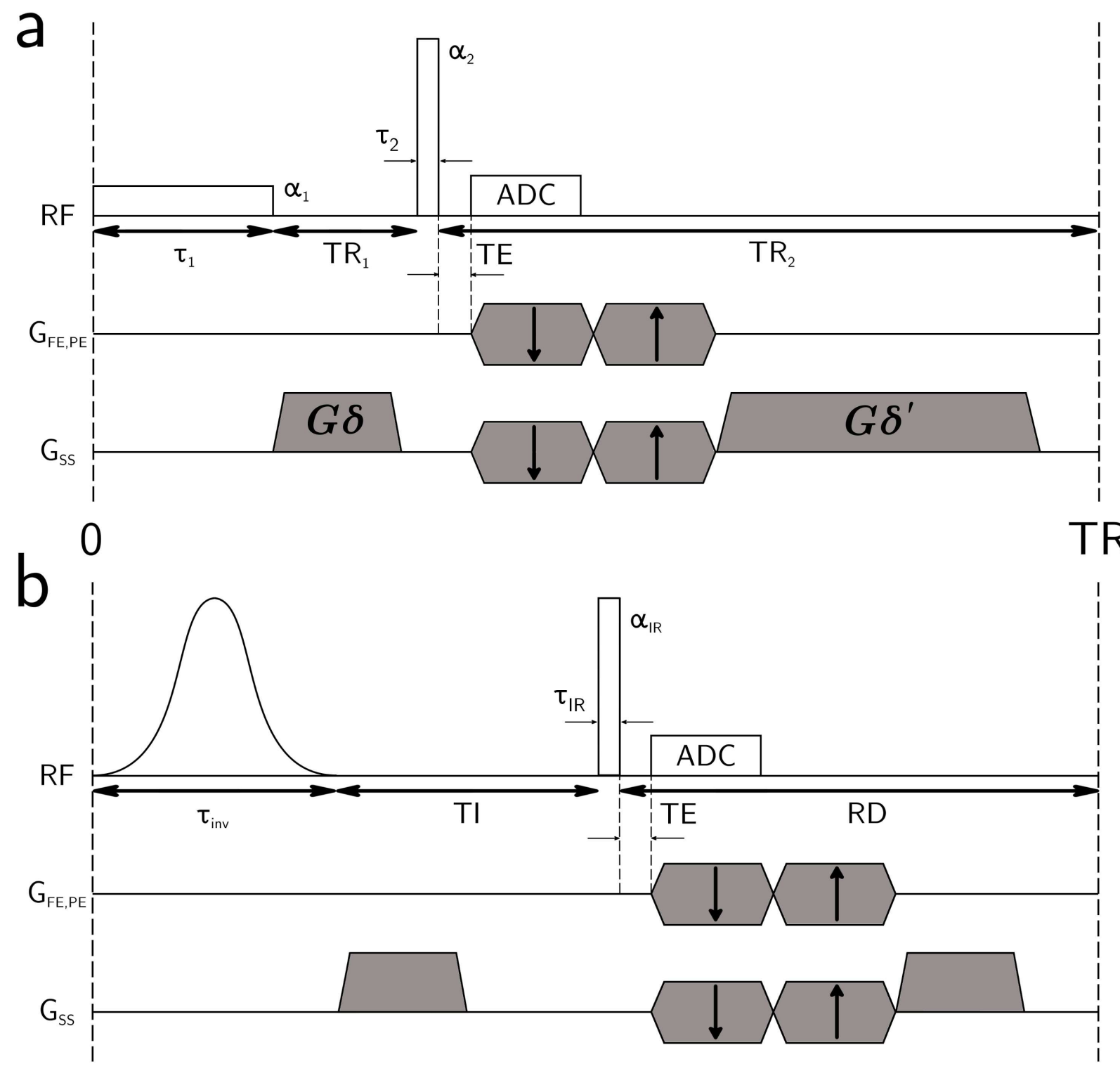

Figure 1: Diff-UTE (a) and IR-UTE (b) pulse sequences. The gradients inducing diffusion weighting in Diff-UTE correspond respectively to the short one after the preparation pulse (relative area $G \delta$ ), and to the long one after the readout gradients refocusing (relative area $\left.G \delta^{\prime}\right)$. 


\begin{tabular}{c|cc} 
Component & Short- $T_{2}$ & Long- $T_{2}$ \\
\hline Parameters & $T_{1}^{S}(\searrow), T_{2}^{S}(\nearrow)$ & $T_{1}^{L}, T_{2}^{L}, \mathrm{D}$ \\
RF Pulses & $\alpha_{1}(\searrow), \alpha_{2}$ & $\alpha_{1}, \alpha_{2}$ \\
& $\tau_{1}(\nearrow), \tau_{2}(\searrow)$ & - \\
Gradients influence & - & $G \delta, G \delta^{\prime}$ \\
Adiabatic inversion effect & saturation & inversion
\end{tabular}

Table 1: Summary of the components' relevant parameters influencing their magnetization. Symbols in parenthesis, if any, indicate the tendencies of the corresponding parameter that would maximize the short- $T_{2}$ component signal in Diff-UTE. 

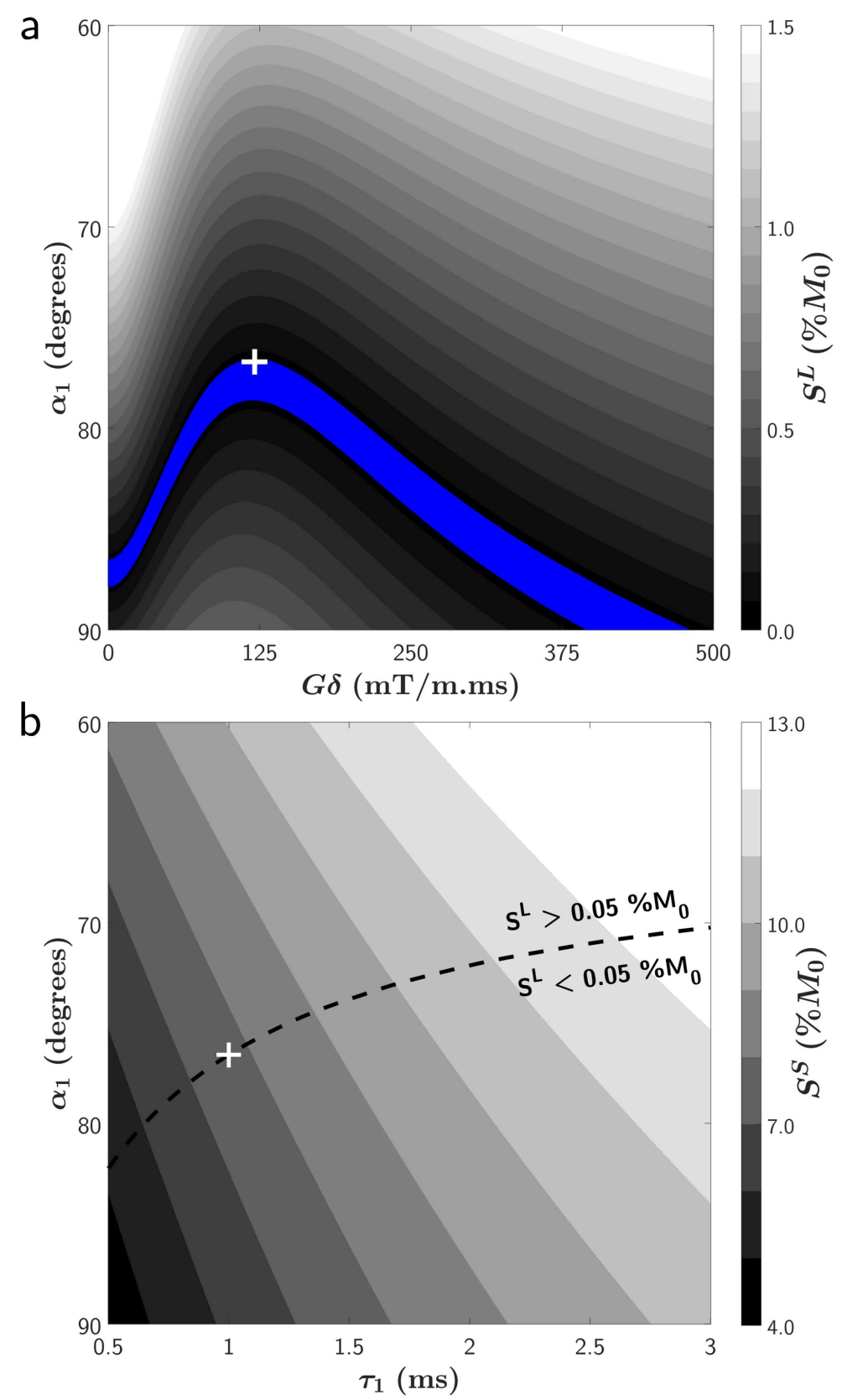

Figure 2: Simulation of long- $T_{2}$ signals along preparation flip angle $\alpha_{1}$ and spoiling gradient moment $G \delta$ for $\tau_{1}=1 \mathrm{~ms}(\mathrm{a})$, and short- $T_{2}$ signals along $\alpha_{1}$ and $\tau_{1}$ (b). In both cases, $\alpha_{2}$ was set to its optimized value given $\alpha_{1}$ and $\tau_{1}$. The dashed line in (b) indicates the lowest $\alpha_{1}$ limit value as a function of $\tau_{1}$ for the proposed optimization scheme to yield a long- $T_{2}$ suppression $\left(S^{L}<0.05 \% M_{0}\right)$, and the white crosses in (a) and (b) relates this aspect for $\tau_{1}=1 \mathrm{~ms}$. Simulation parameters are $T_{1}^{L} / T_{2}^{L}=1000 / 100 \mathrm{~ms}, T_{1}^{S} / T_{2}^{S}=300 / 0.315 \mathrm{~ms}$, $D=1.00 \mu \mathrm{m}^{2} / \mathrm{ms}, \tau_{2}=0.07 \mathrm{~ms}, T R_{1} / T R_{2}=5 / 25 \mathrm{~ms}$. Signal minima occurrences are depicted in the blue bands. 

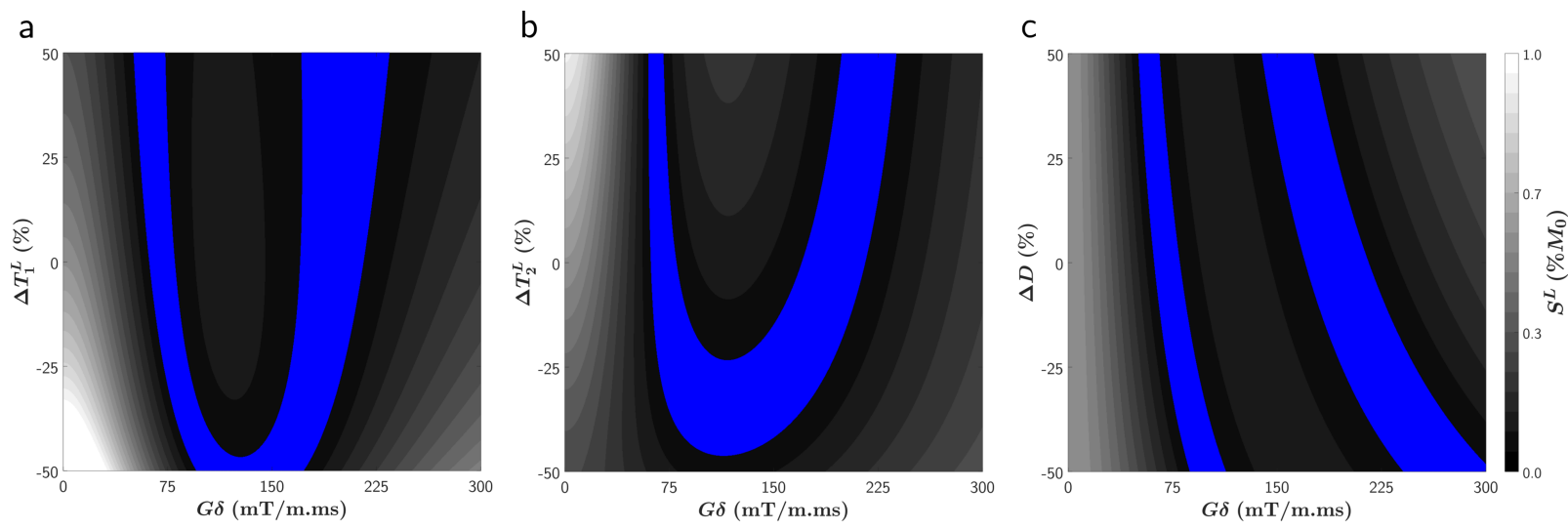

Figure 3: Simulated long- $T_{2}$ signals as functions of spoiling gradient moment and $T_{1}^{L}$ ((a) — nominal $\left.T_{1}^{L}=1000 \mathrm{~ms}\right), T_{2}^{L}\left((\mathrm{~b})-\right.$ nominal $\left.T_{2}^{L}=100 \mathrm{~ms}\right)$ and $D((\mathrm{c})$ - nominal $\left.D=1.00 \mu \mathrm{m}^{2} / \mathrm{ms}\right)$ deviations. Simulation parameters: $T_{1}^{S} / T_{2}^{S}=300 / 0.315 \mathrm{~ms}, \tau_{1} / \tau_{2}=$ $1 / 0.07 \mathrm{~ms}, \alpha_{1} / \alpha_{2}=80 / 57.0^{\circ}$ and $T R_{1} / T R_{2}=5 / 25 \mathrm{~ms}$. Signal minima occurrences $\left(S^{L}<\right.$ $\left.0.05 \% M_{0}\right)$ are depicted in the blue bands. 


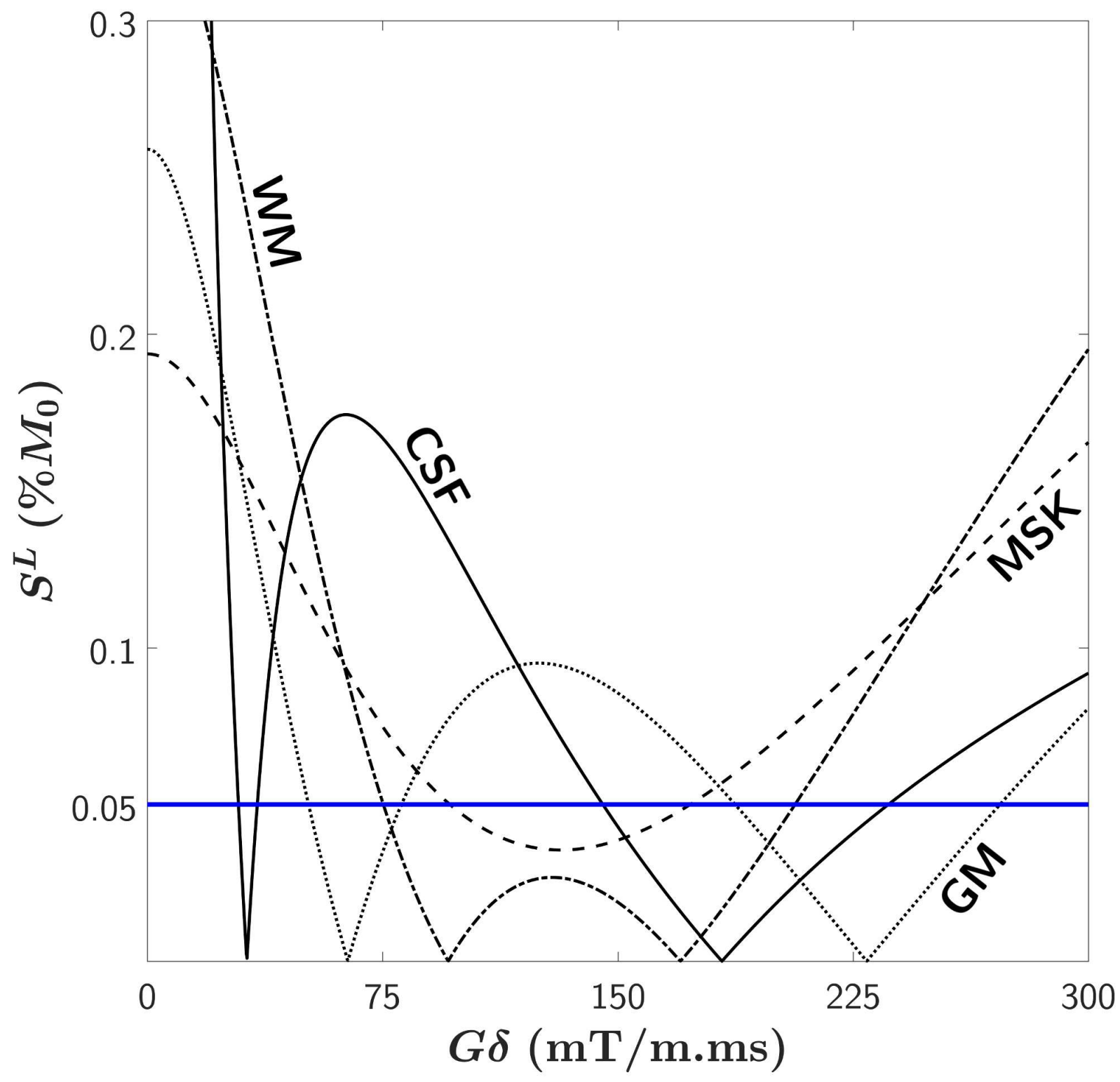

Figure 4: Simulated long- $T_{2}$ signals as functions of spoiling gradient moment for biological tissues: skeletal muscle (MSK, dashed line, $T_{1} / T_{2}=1412 / 50 \mathrm{~ms}, D=0.7 \mu \mathrm{m}^{2} / \mathrm{ms}$ ), cerebrospinal fluid (CSF, solid line, $T_{1} / T_{2}=4000 / 2000 \mathrm{~ms}, D=2.2 \mu \mathrm{m}^{2} / \mathrm{ms}$ ), grey matter (GM, dotted line, $T_{1} / T_{2}=1820 / 99 \mathrm{~ms}, D=0.8 \mu \mathrm{m}^{2} / \mathrm{ms}$ ) and white matter (WM, dashed-dotted line, $\left.T_{1} / T_{2}=1084 / 69 \mathrm{~ms}, D=0.8 \mu \mathrm{m}^{2} / \mathrm{ms}\right)$. Other simulation parameters: $T_{1}^{S} / T_{2}^{S}=300 / 0.315 \mathrm{~ms}, \tau_{1} / \tau_{2}=1 / 0.07 \mathrm{~ms}, \alpha_{1} / \alpha_{2}=80 / 57.0^{\circ}$ and $T R_{1} / T R_{2}=5 / 25 \mathrm{~ms}$. The horizontal blue line indicates the suppression threshold $\left(S^{L}<0.05 \% M_{0}\right)$. 

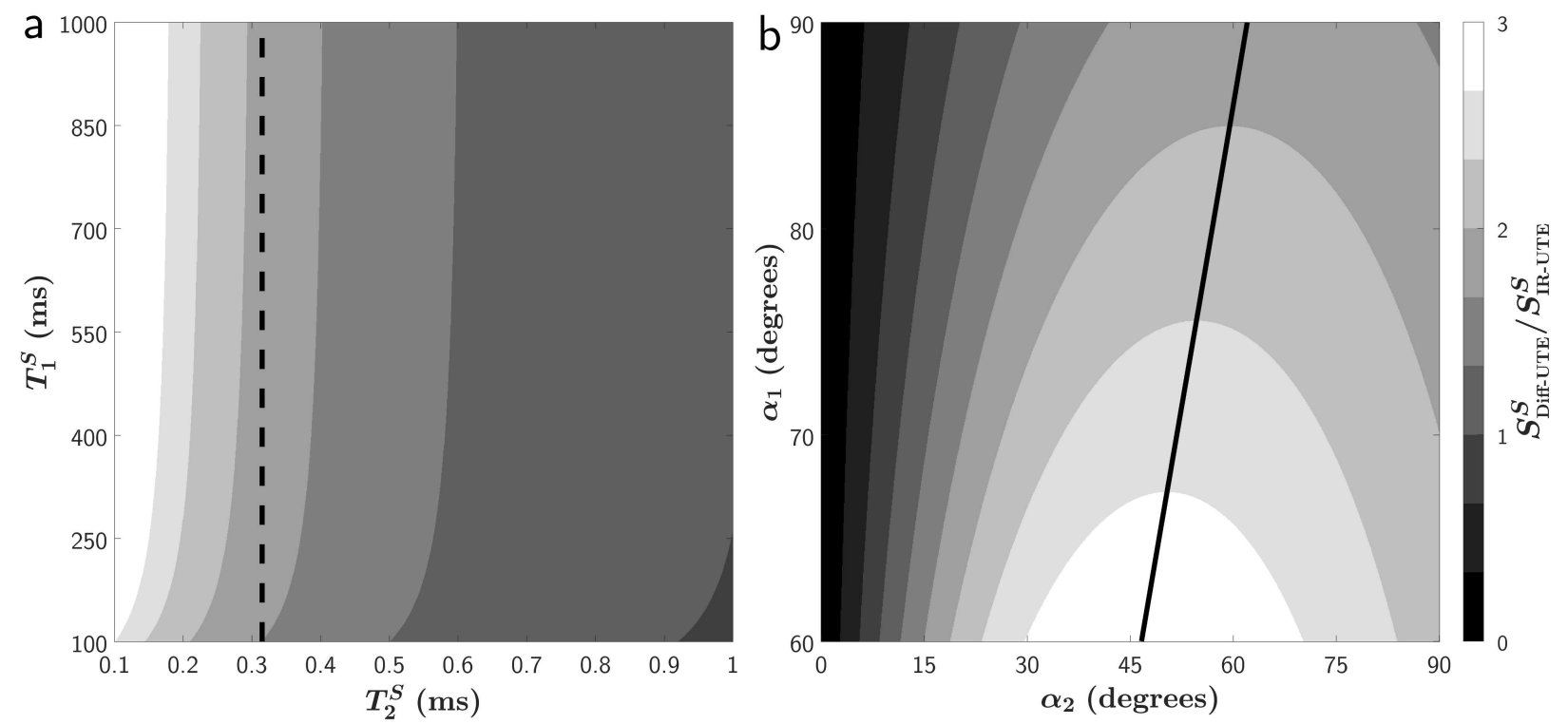

Figure 5: Simulated short- $T_{2}$ signal ratio as a function of $T_{1}^{S}$ and $T_{2}^{S}$ for the most penalizing case of a preparation flip angle $\alpha_{1}=90^{\circ}$ and optimized $\alpha_{2}$ (a), and as a function of $\alpha_{1}$ and $\alpha_{2}$ for $T_{1}^{S} / T_{2}^{S}=300 / 0.315 \mathrm{~ms}$ (b). The black dashed line in (a) corresponds to the estimated $T_{2}^{S}=315 \mu$ s value in the Lego brick. The black solid line in (b) corresponds to the optimized $\alpha_{2}$ maximizing Eq. 11. Other simulation parameters are $\tau_{1} / \tau_{2}=1 / 0.07 \mathrm{~ms}$, and $T R_{1} / T R_{2}=5 / 25 \mathrm{~ms}$ in Diff-UTE, $\tau_{\text {inv }} / \tau_{I R}=8 / 0.07 \mathrm{~ms}$, and $T I / R D=11.5 / 11.5 \mathrm{~ms}$ in IR-UTE (optimized delays to suppress a component having a $T_{1}^{L}$ of $1000 \mathrm{~ms}$ ). 


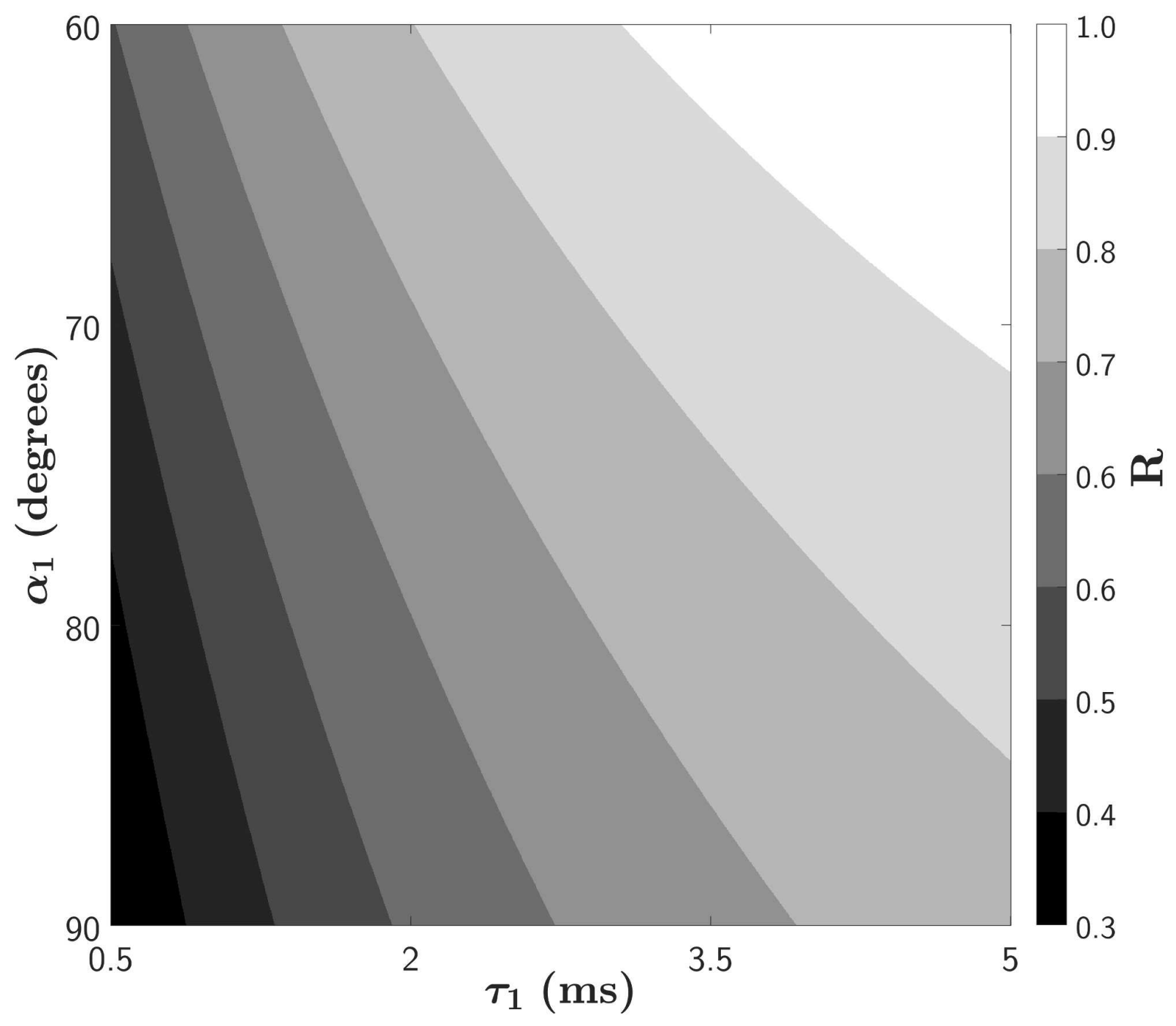

Figure 6: Simulated short- $T_{2}$ signals ratio $R=\left(S_{\text {Diff-UTE }}^{S} / S_{\text {UTE }}^{S}\right) \times \sqrt{2}$ as a function of $\tau_{1}$ and $\alpha_{1}$ for respective optimized $\alpha_{2}$ flip angle. Simulation parameters are $T_{1}^{S} / T_{2}^{S}=300 / 0.315$ $\mathrm{ms}, \tau_{2}=0.07 \mathrm{~ms}$ and $T R_{1} / T R_{2}=5 / 25 \mathrm{~ms}$. 

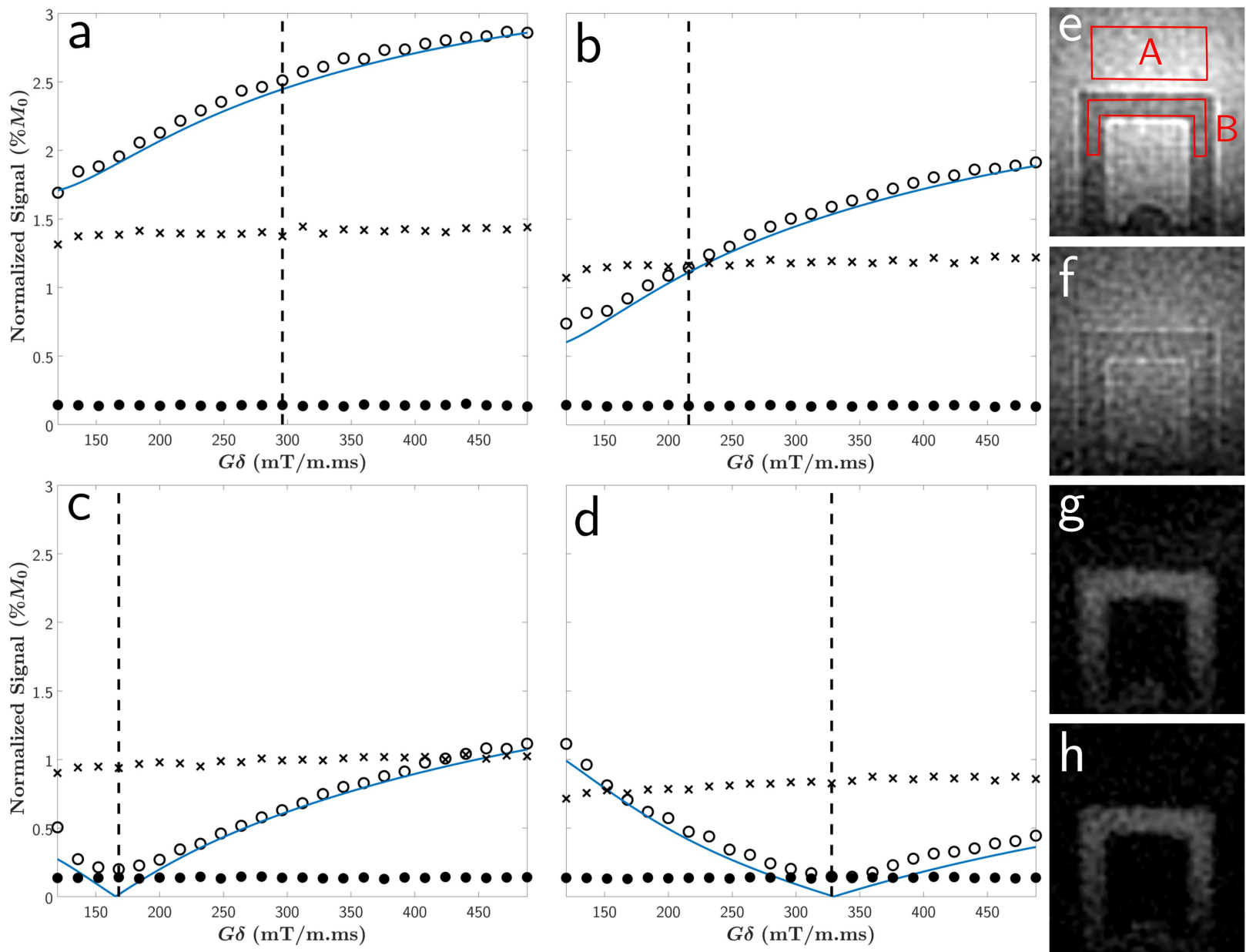

Figure 7: Effect of the Diff-UTE suppression scheme for four preparation flip angle $\alpha_{1}$ values: $\alpha_{1}=60^{\circ}$ (a), $\alpha_{1}=70^{\circ}$ (b), $\alpha_{1}=80^{\circ}$ (c) and $\alpha_{1}=90^{\circ}$ (d) against spoiling gradient moment $G \delta$. Doped-water (circle) and short- $T_{2}$ component (cross) correspond to the mean signal intensities estimated respectively in ROIs A and B drawn in the view (e). The solid line describes the simulated water signal amplitude given the experimental parameters. The noise ROI was drawn away in an artifact-free area (not shown), and its evolution is depicted in bullets. All experimental signals on plots of the different scenarios are normalized to the water signal's intensity corresponding to $\alpha_{1}=60^{\circ}$ and $G \delta=488 \mathrm{mT} / \mathrm{m} . \mathrm{ms}$, the latter being considered to be fully spoiled (the signal evolution being very slow afterwards). Coronal views (e-h) were chosen given relevant spoiling gradient moment values (dashed vertical lines on corresponding plots $\{\mathrm{a}, \mathrm{e}\},\{\mathrm{b}, \mathrm{f}\},\{\mathrm{c}, \mathrm{g}\},\{\mathrm{d}, \mathrm{h}\})$ where the contrast of the short- $T_{2}$ component is either negative (e), null (f) or positive $(\mathrm{g}, \mathrm{h})$, consecutively to the long relaxing component suppression. 


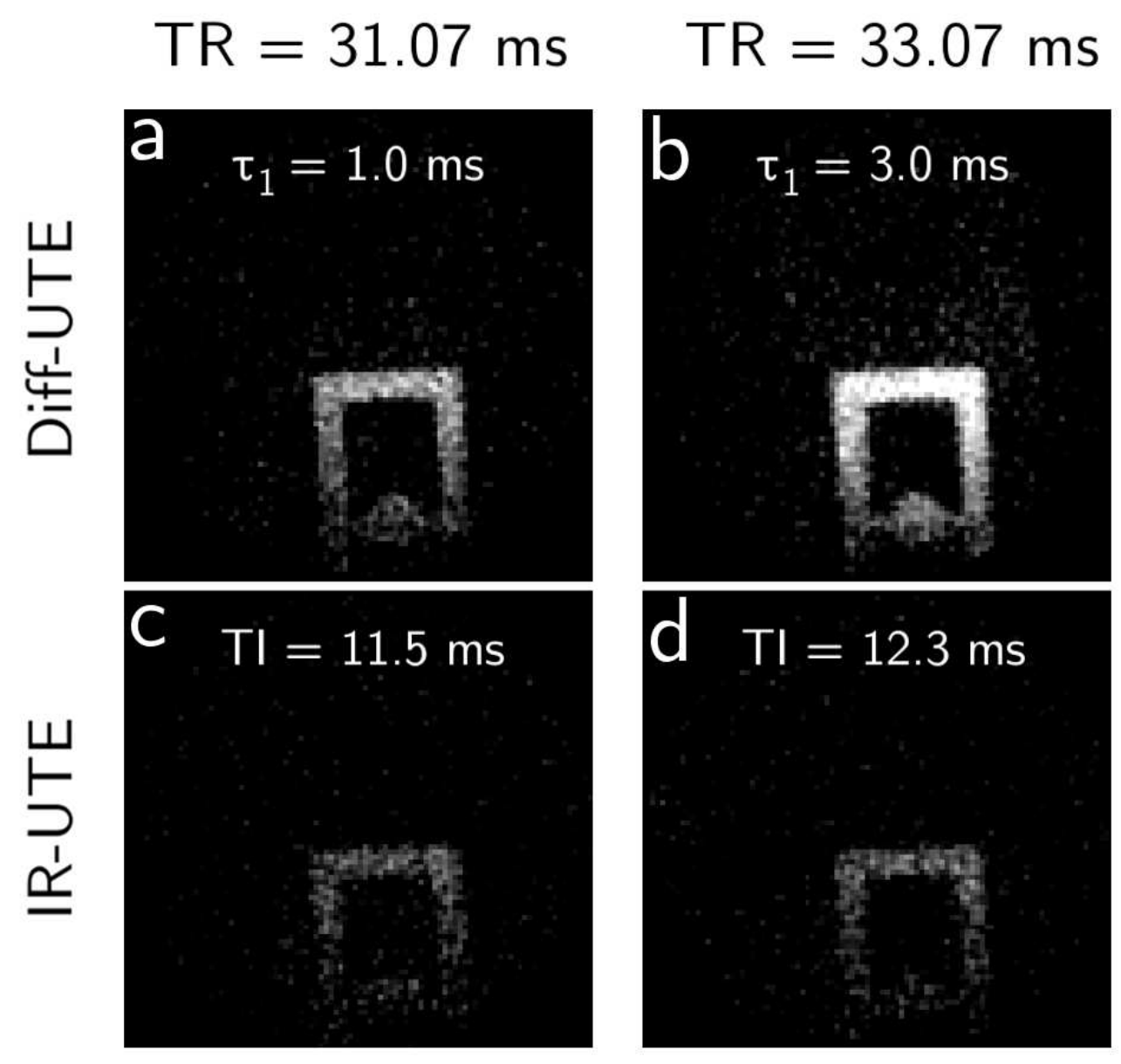

Figure 8: Coronal slices acquired in Diff-UTE for $\tau_{1}$ of 1.0 (a) and $3.0 \mathrm{~ms}$ (b) in Diff-UTE, and for $T I$ of 11.5 (c) and $12.3 \mathrm{~ms}$ (d) in IR-UTE. The same contrast window levels is used in each view. $T R$ is kept constant in both sequences in each column $\{\mathrm{a}, \mathrm{c}\}$ and $\{\mathrm{b}, \mathrm{d}\}$. Experimental SNR ratios of the short- $T_{2}$ component generated between Diff-UTE and IRUTE amount to 1.49 for $T R=31.07 \mathrm{~ms}$, and 2.31 for $T R=33.07 \mathrm{~ms}$. 

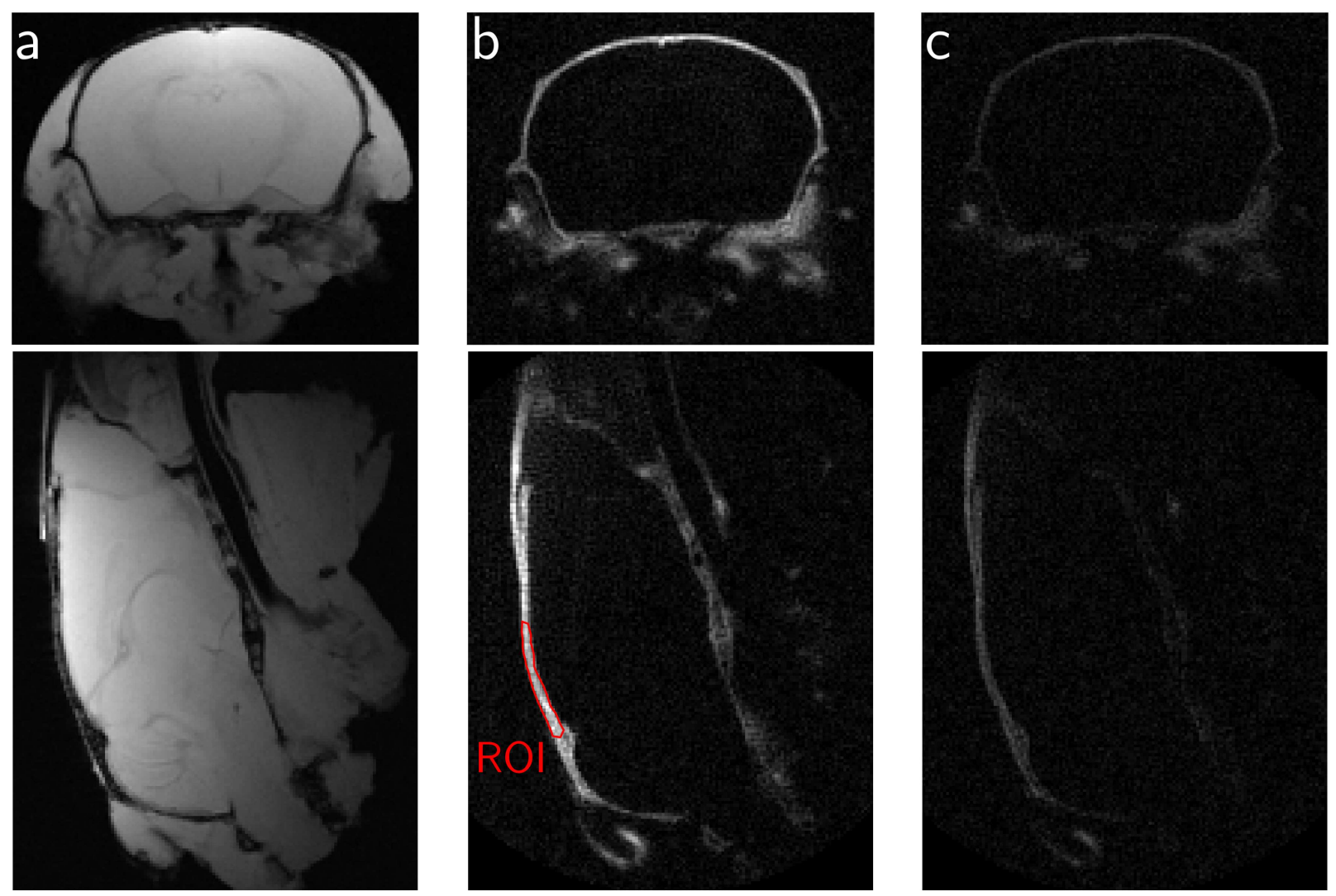

Figure 9: Coronal (top row) and sagittal (bottom row) views of the fixed mouse head in FLASH (a), Diff-UTE (b) and IR-UTE (c). The same dynamic range was used in all four UTE images. The bone ROI used for SNR analysis between Diff-UTE and IR-UTE is drawn in red on the sagittal view of the Diff-UTE column (b). The noise ROI (not shown) was drawn far from the head in an artifact-free area. 